\title{
The Master Ward Identity for Scalar QED
}

\author{
Michael Dütsch, Luis Peters and Karl-Henning Rehren®()
}

\begin{abstract}
It is emphasized that for interactions with derivative couplings, the Ward Identity (WI) securing the preservation of a global symmetry should be modified. Scalar QED is taken as an explicit example. More precisely, it is rigorously shown in scalar QED that the naive WI and the improved Ward Identity ('Master Ward Identity', MWI) are related to each other by a finite renormalization of the time-ordered product (' $T$ product') for the derivative fields, and we point out that the MWI has advantages over the naive WI-in particular with regard to the proof of the MWI. We show that the MWI can be fulfilled in all orders of perturbation theory by an appropriate renormalization of the $T$-product, without conflict with other standard renormalization conditions. Relations with other recent formulations of the MWI are established.
\end{abstract}

\section{Introduction}

In spinor QED, the Master Ward Identity (MWI) expressing global $U(1)$ symmetry contains all information that is needed for a consistent perturbative BRST-construction of the model, see [8] or [6, Chap. 5]. This 'QED-MWI' is a renormalization condition on $T$-products ${ }^{1}$ to be satisfied to all orders of perturbation theory. It reads

$$
\begin{aligned}
& \partial_{y}^{\mu} T_{n+1}\left(\widetilde{B}_{1}\left(x_{1}\right) \otimes \cdots \otimes \widetilde{B}_{n}\left(x_{n}\right) \otimes j_{\mu}(y)\right)_{0} \\
& \quad=-\sum_{l=1}^{n} \delta\left(y-x_{l}\right) T_{n}\left(\widetilde{B}_{1}\left(x_{1}\right) \otimes \cdots \otimes \widetilde{\theta B_{l}}\left(x_{l}\right) \otimes \cdots \otimes \widetilde{B}_{n}\left(x_{n}\right)\right)_{0},
\end{aligned}
$$

where $j^{\mu}=\bar{\psi} \gamma^{\mu} \psi$ is the Dirac current, $B_{1}, \ldots, B_{n}$ are arbitrary submonomials (see (A.3)) of the interaction $L=e j^{\mu} A_{\mu}$, and $\theta$ is the charge number operator.

\footnotetext{
${ }^{1}$ We understand the term 'renormalization condition' in the precise sense of the inductive Epstein-Glaser construction of $T$-products [12]: a constraint on extensions of distributions, as explained in Appendix A.1.
} 
The notation $\widetilde{(\cdot)}$ means that fermionic field polynomials are converted into bosonic field polynomials by multiplying them with a Grassmann variable. By $T(\ldots)_{0}$, we denote on-shell $T$-products (see below).

There is an essential difference between spinor QED and scalar QED: in the latter, the current to which the electromagnetic potential is coupled, contains first derivatives of the basic fields: ${ }^{2}$

$$
j^{\mu}:=i\left(\phi \partial^{\mu} \phi^{*}-\phi^{*} \partial^{\mu} \phi\right) .
$$

It is apriori not evident how to translate the QED-MWI (1.1) to models with derivative couplings, and scalar QED may serve as a prototype of such models.

Our results can be summarized as follows: in Sect. 2, we postulate a naive WI for scalar QED, just by analogy to spinor QED. To fulfil it, an 'unnatural' renormalization of the $T$-product of $\partial^{\mu} \phi(x)$ with $\partial^{\nu} \phi^{*}(y)$ is required [11]: one has to add $i g^{\mu \nu} \delta(x-y)$ to $\partial^{\nu} \partial^{\mu} \Delta^{F}(x-y)$. This addition violates the standard renormalization conditions 'Field Equation' and 'Action Ward Identity'.

In Sect. 3, we work out the MWI for the global $U(1)$-transformation $\phi(x) \rightarrow e^{i \alpha} \phi(x)$ in scalar QED, and find that compared with the naive WI, it contains an additional term.

In Sect. 5, we prove that the MWI can be fulfilled by an appropriate renormalization of the $T$-product, which is compatible with the further standard renormalization conditions.

In Sect. 6, starting with the time-ordered product ' $T$ ' we define in all orders a new time-ordered product $\widehat{T}$ induced from the initial finite renormalization $\partial^{\nu} \partial^{\mu} \Delta^{F} \rightarrow \partial^{\nu} \partial^{\mu} \Delta^{F}+i g^{\mu \nu} \delta$, by the inductive Epstein-Glaser method [12]. We prove that the validity of the MWI for $T$ is equivalent to the validity of naive WI for $\widehat{T}$. In fact, one may continuously interpolate between $T$ and $\widehat{T}$.

In Sect. 4.2, we prove, in the perturbative approach to scalar QED, that the MWI is equivalent to the so-called 'unitary MWI'. The latter is an identity, conjectured by Fredenhagen [4], which seems to be well-suited for the formulation of symmetries in the Buchholz-Fredenhagen quantum algebra [5].

All proofs are given to all orders of perturbation theory.

\subsection{Some Technical Preparations}

We use natural units, in particular $\hbar=1$, and the underlying spacetime is the 4-dimensional Minkowski space $\mathbb{M}$. We work with causal perturbation theory, also called 'Epstein-Glaser method' [12]. This method is based on an axiomatic definition of the time-ordered product $T \equiv\left(T_{n}\right)_{n=1}^{\infty}$, the most important axiom being a causal factorization property of $T_{n}$ (see Appendix A.1), and yields an inductive construction of the sequence $\left(T_{n}\right)$ solving the axioms. In addition, we

\footnotetext{
${ }^{2}$ This is the Noether current pertaining to the invariance of the free action of the scalar field under the global $U(1)$-transformation $\phi(x) \rightarrow e^{i \alpha} \phi(x)(\alpha \in \mathbb{R})$. The Dirac current is defined w.r.t. $\psi \rightarrow e^{-i \alpha} \psi$. This switch of sign convention will explain a number of opposite signs in the present formulas as compared to spinor QED in [6], notably (2.2) and (3.2).
} 
use the formalism where quantum fields are functionals on classical configuration spaces, equipped with a non-commutative product: the star product of the free theory (denoted by ' $\star$ ', see (1.5)). Perturbation theory represents interacting fields as formal power series within this algebra, using the time-ordered product of local fields, which is commutative. The prominent mathematical task is the construction of the time-ordered product. For details and conventions, we refer to the book [6], where in particular the conventions for the propagators are fixed in [6, App. A.2].

For the convenience of the reader, we sketch some basic definitions of the formalism for the model at hand, that is, scalar QED. The expert reader may skip the remainder of this section, except for the third and second last paragraph containing some remarks about 'on-shell MWI versus off-shell MWI' and the definition of $\mathcal{P}$. The basic fields of scalar QED are a complex scalar field $\phi(x)$, its conjugate field $\phi^{*}(x)$ and the photon field $A(x) \equiv\left(A^{\mu}(x)\right)$. The configuration space is $\mathcal{C}=C^{\infty}(\mathbb{M}, \mathbb{C}) \times C^{\infty}\left(\mathbb{M}, \mathbb{R}^{4}\right)$, where the first factor stands for the configurations of $\phi, \phi^{*}$ and the second for the configurations of $\left(A^{\mu}\right)$. The basic fields are the evaluation functionals

$$
\begin{aligned}
\phi(x)[h] & =h(x), \quad \phi^{*}(x)[h]=\overline{h(x)}, \quad A^{\mu}(x)[a]=a^{\mu}(x), \\
\forall h & \in C^{\infty}(\mathbb{M}, \mathbb{C}), a \equiv\left(a_{\mu}\right) \in C^{\infty}\left(\mathbb{M}, \mathbb{R}^{4}\right),
\end{aligned}
$$

where the overline denotes complex conjugation. The space of fields $\mathcal{F}$ is the set of all polynomial functionals on the configuration space satisfying certain properties. More precisely, a field $\mathcal{F} \ni F: \mathcal{C} \rightarrow \mathbb{C}$ is a finite sum of functionals of the form

$$
\begin{aligned}
F= & \sum_{p, n, l} \int d x_{1} \cdots d x_{p} d y_{1} \cdots d y_{n} d z_{1} \cdots d z_{l} \prod_{i=1}^{p} A_{\mu_{i}}\left(x_{i}\right) \prod_{j=1}^{n} \phi\left(y_{j}\right) \prod_{k=1}^{l} \phi^{*}\left(z_{k}\right) \\
& \cdot f_{p, n, l}^{\mu_{1} \ldots \mu_{p}}\left(x_{1}, \ldots, x_{p}, y_{1}, \ldots, y_{n}, z_{1}, \ldots, z_{l}\right) \\
= & : \sum_{p, n, l}\left\langle f_{p, n, l}^{\mu_{1} \ldots \mu_{p}},\left(\otimes_{i=1}^{p} A_{\mu_{i}}\right) \otimes \phi^{\otimes n} \otimes\left(\phi^{*}\right)^{\otimes l}\right\rangle,
\end{aligned}
$$

evaluated as

$$
F[h, a]:=\sum_{p, n, l}\left\langle f_{p, n, l}^{\mu_{1} \ldots \mu_{p}},\left(\otimes_{i=1}^{p} a_{\mu_{i}}\right) \otimes h^{\otimes n} \otimes(\bar{h})^{\otimes l}\right\rangle \quad \forall(h, a) \in \mathcal{C},
$$

where $f_{0,0,0} \in \mathbb{C}$ is constant; and for $p+n+l \geq 1$, each expression $f_{p, n, l}^{\mu_{1} \ldots}$ is an element of $\mathcal{D}^{\prime}\left(\mathbb{M}^{p+n+l}, \mathbb{C}\right)$ with compact support, which satisfies a certain wave front set condition (not relevant in this work, see [6, Def. 1.2.1]). The purpose of the latter is to ensure the existence of the pointwise products of distributions appearing in the definition of the star product (1.5).

The support of $F \in \mathcal{F}$ is defined by

$$
\operatorname{supp} F:=\overline{\bigcup_{(h, a) \in \mathcal{C}} \bigcup_{\varphi=A^{\mu}, \phi, \phi^{*}} \operatorname{supp} \frac{\delta F}{\delta \varphi(\cdot)}[h, a]},
$$

where on the r.h.s. we mean the support in the sense of distributions. 
Convergence in $\mathcal{F}$ is understood in the pointwise sense: $\lim _{n \rightarrow \infty} F_{n}=F$ if and only if $\lim _{n \rightarrow \infty} F_{n}[h, a]=F[h, a]$ for all $(h, a) \in \mathcal{C}$ (cf. [6, formula $(1.2 .3)])$. For example, the closure on the r.h.s. of (1.4) is done in this sense; or, by $\mathcal{D}^{\prime}(\mathbb{M}, \mathcal{F})$ we mean the vector space of all linear maps from $\mathcal{D}(\mathbb{M})$ to $\mathcal{F}$, which are continuous w.r.t. this topology on $\mathcal{F}$.

The vacuum expectation value $(\mathrm{VEV})$ of a field $F \in \mathcal{F}$ is $\omega_{0}(F):=F[0,0]$.

For the complex scalar field, the free field equation is the Klein-Gordon equation: $\left(\square+m^{2}\right) \phi(x)=0=\left(\square+m^{2}\right) \phi(x)^{*}$. For the photon field we use the Feynman gauge, that is, the free field equation is the wave equation $\square A^{\mu}(x)=$ 0 ; see [6, Sect. 5.1.3].

The space of fields $\mathcal{F}$ is equipped with the following operations:

- An involutive *-operation, defined on the elements (1.3) by

$$
F^{*}:=\sum_{p, n, l}\left\langle\overline{f_{p, n, l}^{\mu_{1} \ldots \mu_{p}}},\left(\otimes_{i=1}^{p} A_{\mu_{i}}\right) \otimes\left(\phi^{*}\right)^{\otimes n} \otimes \phi^{\otimes l}\right\rangle
$$

(again the overline denotes complex conjugation), in particular $\phi$ and $\phi^{*}$ are mutually exchanged;

- the pointwise or classical product,

$(F \cdot G)[h, a]:=F[h, a] \cdot G[h, a], \quad \forall F, G \in \mathcal{F},(h, a) \in C^{\infty}(\mathbb{M}, \mathbb{C}) \times C^{\infty}\left(\mathbb{M}, \mathbb{R}^{4}\right)$, which is commutative.

- The free theory is quantized by deforming the classical product into a noncommutative product - the star product; to simplify the notations, we give here the definition for the model of one real scalar field $\varphi$ :

$$
\begin{aligned}
F \star G:= & \sum_{n=0}^{\infty} \frac{\hbar^{n}}{n !} \int d x_{1} \cdots d x_{n} d y_{1} \cdots d y_{n} \\
& \frac{\delta^{n} F}{\delta \varphi\left(x_{1}\right) \cdots \delta \varphi\left(x_{n}\right)} \prod_{l=1}^{n} \Delta_{m}^{+}\left(x_{l}-y_{l}\right) \frac{\delta^{n} G}{\delta \varphi\left(y_{1}\right) \cdots \delta \varphi\left(y_{n}\right)},
\end{aligned}
$$

where $\Delta_{m}^{+}$is the Wightman two-point function to the mass $m$. Exceptionally, we write here $\hbar$, because it is the deformation parameter. More precisely, the star product is a map $\mathcal{F} \llbracket \hbar \rrbracket \times \mathcal{F} \llbracket \hbar \rrbracket \rightarrow \mathcal{F} \llbracket \hbar \rrbracket$, where $\mathcal{F} \llbracket \hbar \rrbracket$ denotes the space of formal power series in $\hbar$ with coefficients in $\mathcal{F}$. When (1.5) is adapted to scalar QED, the only non-vanishing 'contractions' in the definition of the star product are

$$
\begin{aligned}
& \omega_{0}\left(\phi^{*}(x) \star \phi(y)\right)=\hbar \Delta_{m}^{+}(x-y)=\omega_{0}\left(\phi(x) \star \phi^{*}(y)\right), \\
& \omega_{0}\left(A^{\mu}(x) \star A^{\nu}(x)\right)=-\hbar g^{\mu \nu} D^{+}(x-y),
\end{aligned}
$$

where $D^{+}:=\Delta_{m=0}^{+}$.

To a far extent, we work on-shell. This means that all functionals $F \in \mathcal{F}$ are restricted to the space $\mathcal{C}_{S_{0}}$ of solutions of the free field equations; we indicate this restriction by

$$
F_{0}:=\left.F\right|_{\mathcal{C}_{S_{0}}} \quad \forall F \in \mathcal{F}
$$


Algebraically, on-shell fields can be identified with Fock space operators, where the star product of on-shell fields corresponds to the operator product, and the pointwise product of on-shell functionals (i.e. $\left(F_{0} \cdot G_{0}\right)[h, a]:=F_{0}[h, a] \cdot G_{0}[h, a]$ for all $F, G \in \mathcal{F}$ and $\left.(h, a) \in \mathcal{C}_{S_{0}}\right)$ to the normally ordered product, see $[6$, Thm. 2.6.3]. The motivations to study only the on-shell version of the MWI in this paper are the following: firstly, the essential information of the off-shell MWI is already contained in its on-shell version (see Remark 3.1); secondly, working in Fock space one 'sees' only the on-shell MWI; and finally, off-shell notation would just unnecessarily overburden many formulas.

Throughout this paper, we need only distributions $f_{p, n, l}^{\mu_{1} \ldots}$ such that no derivatives of $A^{\mu}$ and solely zeroth and first derivatives of $\phi$ and $\phi^{*}$ appear. So we define $\mathcal{P}$ to be the space of polynomials in $A^{\mu}, \phi, \phi^{*}, \partial^{\mu} \phi$ and $\partial^{\nu} \phi^{*}$ only.

The subspace $\mathcal{F}_{\text {loc }} \subset \mathcal{F}$ of local fields is the linear span of the set $\left\{B(g) \equiv \int d x g(x) B(x) \mid B \in \mathcal{P}, g \in \mathcal{D}(\mathbb{M})\right\}$. For example:

$$
\left(\partial^{\mu} \phi^{*} \partial_{\mu} \phi\right)(g)[h, a]=\int d x g(x) \partial^{\mu} \overline{h(x)} \partial_{\mu} h(x), \quad \forall(h, a) \in \mathcal{C} .
$$

\section{The Naive Ward Identity}

A natural candidate for the Ward Identity (WI) expressing global $U(1)$-symmetry for scalar QED just copies the QED-MWI (1.1) with the charge number operator

$$
\theta B:=\phi \frac{\partial B}{\partial \phi}+\partial^{\mu} \phi \frac{\partial B}{\partial\left(\partial^{\mu} \phi\right)}-\phi^{*} \frac{\partial B}{\partial \phi^{*}}-\partial^{\mu} \phi^{*} \frac{\partial B}{\partial\left(\partial^{\mu} \phi^{*}\right)} \quad \text { for } B \in \mathcal{P},
$$

and with the time-ordered product $\widehat{T}$ that is required to satisfy the basic axioms (i)-(iv) and the renormalization conditions (v)-(viii) listed in Appendix A.1. This yields

$$
\begin{aligned}
\partial_{y}^{\mu} & \widehat{T}_{n+1}\left(B_{1}\left(x_{1}\right) \otimes \cdots \otimes B_{n}\left(x_{n}\right) \otimes j_{\mu}(y)\right)_{0} \\
& =\sum_{l=1}^{n} \delta\left(y-x_{l}\right) \widehat{T}_{n}\left(B_{1}\left(x_{1}\right) \otimes \cdots \otimes\left(\theta B_{l}\right)\left(x_{l}\right) \otimes \cdots \otimes B_{n}\left(x_{n}\right)\right)_{0},
\end{aligned}
$$

where $B_{1}, \ldots, B_{n}$ are arbitrary submonomials of the interaction

$$
\widetilde{L}:=e j^{\mu} A_{\mu} \text {. }
$$

This Ward identity is a generalization of the one postulated and proved in [11]; the difference is that in this reference only the neutral fields $B_{1}, \ldots, B_{n} \in$ $\left\{\widetilde{L}, j^{\mu}\right\}$ are studied and, hence, the r.h.s. of (2.2) vanishes. In [11], the WI there is motivated by gauge invariance of the on-shell $S$-matrix (i.e. the restriction to $\mathcal{C}_{S_{0}}$ of the $S$-matrix defined in (A.4)), that is, invariance under the transformation $A^{\mu}(x) \rightarrow A^{\mu}(x)+\partial^{\mu} \Lambda(x)$ of $\widehat{\mathbf{S}}\left(g, e j^{\mu} A_{\mu}\right)_{0}$ in the formal adiabatic limit $g(x) \rightarrow 1 \forall x$. The task would be to establish the existence of $\widehat{T}$ satisfying (i)-(viii) and (2.2). 
In Sect. 3, we show that (2.2) is only a simplified version of the Master Ward Identity (MWI) expressing $U(1)$-symmetry; the latter is better suited for models with derivative couplings, and is easier to establish.

Particular cases of the WI (2.2) are

$$
\begin{aligned}
& \partial_{\mu}^{y} \widehat{T}\left(j^{\nu}(x) \otimes j^{\mu}(y)\right)_{0}=0, \quad \partial_{\mu}^{y} \widehat{T}\left(\partial^{\nu} \phi(x) \otimes j^{\mu}(y)\right)_{0}=\delta(y-x) \partial^{\nu} \phi(x)_{0}, \\
& \partial_{\mu}^{y} \widehat{T}\left(\partial^{\nu} \phi^{*}(x) \otimes j^{\mu}(y)\right)_{0}=-\delta(y-x) \partial^{\nu} \phi^{*}(x)_{0} .
\end{aligned}
$$

These identities have an important property: Requiring that $\widehat{T}$ satisfies the axiom (v) Field Independence that is, the validity of the causal Wick expansion (A.2), the tree diagram part of the first identity, and the other two identities are fulfilled if and only if the numerical distribution $\hat{t}\left(\partial^{\nu} \phi, \partial^{\mu} \phi^{*}\right)=\omega_{0}\left(\widehat{T}\left(\partial^{\nu} \phi \otimes\right.\right.$ $\left.\left.\partial^{\mu} \phi^{*}\right)\right)($ cf. (A.5)) is specified as

$\hat{t}\left(\partial^{\nu} \phi, \partial^{\mu} \phi^{*}\right)(x-y)=-\partial^{\nu} \partial^{\mu} \Delta^{F}(x-y)-i g^{\mu \nu} \delta(x-y)=\hat{t}\left(\partial^{\nu} \phi^{*}, \partial^{\mu} \phi\right)(x-y)$,

as one sees by explicit computation. The finite renormalization of the Feynman propagator with two derivatives

$$
\partial^{\nu} \partial^{\mu} \Delta^{F}(x-y) \longmapsto \partial^{\nu} \partial^{\mu} \Delta^{F}(x-y)+i g^{\mu \nu} \delta(x-y)
$$

is admissible in the framework of causal perturbation theory, since the singular order is $\omega\left(\partial^{\nu} \partial^{\mu} \Delta^{F}\right)=0$ (see (A.7) for the definition of the singular order).

The additional term $i g^{\mu \nu} \delta(x-y)$ has the advantage, that it generates as a necessary finite 'counter term' the quartic interaction part, i.e. $e^{2} A^{\mu} A_{\mu} \phi \phi^{*}$ (as it was first realized in [11]), propagating correctly to higher orders in the inductive Epstein-Glaser construction of $\widehat{T} \equiv\left(\widehat{T}_{n}\right)$. Indeed, for the $S$-matrix belonging to $\widehat{T}$ (defined in (A.4)) we obtain

$$
\begin{aligned}
& \widehat{\mathbf{S}}(g, e j A)= 1+i e(j A)(g) \\
&-\frac{e^{2}}{2} \int d x d y g(x) g(y) \\
& {\left[\hat{t}\left(\partial^{\mu} \phi^{*}, \partial^{\nu} \phi\right)(x-y) A_{\mu}(x) \phi(x) A_{\nu}(y) \phi^{*}(y)+\left(\phi \leftrightarrow \phi^{*}\right)\right]+\ldots } \\
&=1+i\left(e(j A)(g)+e^{2}\left(A A \phi^{*} \phi\right)\left(g^{2}\right)\right)+\ldots,
\end{aligned}
$$

where the dots contain further terms of order $\mathcal{O}\left((e g)^{2}\right)$ and all terms of higher orders in $(e g)$.

But the addition $i g^{\mu \nu} \delta(x-y)$ has the disadvantages that it violates the renormalization condition 'Field Equation' (FE) and the 'Action Ward Identity' (AWI) (generally formulated in Appendix A.1):

$$
\begin{aligned}
& \text { FE: } \quad \hat{t}\left(\partial^{\nu} \phi, \partial^{\mu} \phi^{*}\right)(x-y) \neq \int d z \partial^{\nu} \Delta^{F}(x-z) \frac{\delta \partial^{\mu} \phi^{*}(y)}{\delta \phi^{*}(z)}\left(=-\partial^{\nu} \partial^{\mu} \Delta^{F}(x-y)\right), \\
& \text { AWI: } \hat{t}\left(\partial^{\nu} \phi, \partial^{\mu} \phi^{*}\right)(x-y) \neq \partial_{x}^{\nu} \partial_{y}^{\mu} \hat{t}\left(\phi, \phi^{*}\right)(x-y)\left(=-\partial^{\nu} \partial^{\mu} \Delta^{F}(x-y)\right) .
\end{aligned}
$$

A proof of the WI (2.2) along the lines of the proof of the QED-MWI in [6, Chap. 5.2.2] would require additional work, because that proof uses essentially that the time-ordered product fulfils the 'Field Equation'. Instead, an indirect proof via the MWI is given in Sect. 6. 


\section{The Master Ward Identity}

Due to the mentioned bad properties of the time-ordered product $\widehat{T}$ and the resulting problems in trying to adapt the proof of the QED-MWI to the WI (2.2), we prefer to work with the complete relevant MWI for scalar QED.

The original references for the MWI are [7,9] and [2]. It is a universal formulation of symmetries; it can be understood as the straightforward generalization to QFT of the most general classical identity for local fields that can be obtained from the field equation and the fact that classical fields may be multiplied pointwise. In contrast, the quantum version of the MWI is a renormalization condition with regard to the axioms for the $T$-product (cf. Appendix A.1). It cannot always be fulfilled due to the well-known anomalies.

\subsection{Working Out the Relevant MWI for Scalar QED}

Generally, the on-shell MWI (see [2,9] and [6, Chap. 4.2]) is derived from the symmetry at hand. It reads

$$
\begin{aligned}
T_{n+1} & \left(B_{1}\left(x_{1}\right) \otimes \cdots \otimes B_{n}\left(x_{n}\right) \otimes \delta_{Q(y)} S_{0}\right)_{0} \\
& =i \sum_{l=1}^{n} T_{n}\left(B_{1}\left(x_{1}\right) \otimes \cdots \otimes \delta_{Q(y)} B_{l}\left(x_{l}\right) \otimes \cdots \otimes B_{n}\left(x_{n}\right)\right)_{0},
\end{aligned}
$$

where $\delta_{Q(y)}$ is a functional differential operator specified by the symmetry, and the time-ordered product $T$ is required to fulfil the axioms (i)-(viii) given in Appendix A.1 and the additional renormalization conditions AWI and FE.

In the case at hand, we study the global $U(1)$-transformation $\phi(y) \rightarrow$ $e^{i \alpha} \phi(y)(\alpha \in \mathbb{R})$. Let

$$
Q(y):=-\left.\frac{d}{d \alpha}\right|_{\alpha=0} e^{i \alpha} \phi(y)=-i \phi(y)
$$

and the pertinent functional differential operator

$$
\delta_{Q(y)}:=Q(y) \frac{\delta}{\delta \phi(y)}+Q^{*}(y) \frac{\delta}{\delta \phi^{*}(y)} .
$$

Introduce a modification $\theta_{\mu}$ of the charge number operator,

$$
\theta_{\mu} B:=\phi \frac{\partial B}{\partial\left(\partial^{\mu} \phi\right)}-\phi^{*} \frac{\partial B}{\partial\left(\partial^{\mu} \phi^{*}\right)} \quad \text { for } B \in \mathcal{P},
$$

and recall that ${ }^{3}$

$$
S_{0}:=\int d x\left(\partial_{\mu} \phi^{*}(x) \partial^{\mu} \phi(x)-m^{2} \phi^{*}(x) \phi(x)\right)+S_{0}\left(A^{\mu}\right) .
$$

Then, one verifies straightforwardly that

$\delta_{Q(y)} S_{0}=\partial_{\mu} j^{\mu}(y), \quad \delta_{Q(y)} B(x)=-i\left(\delta(y-x)(\theta B)(x)-\partial_{y}^{\mu}\left(\delta(y-x)\left(\theta_{\mu} B\right)(x)\right)\right)$.

\footnotetext{
${ }^{3} S_{0}$ is a formal expression that is not an element of $\mathcal{F}$, because the configurations are not necessarily vanishing at infinity. Only functional derivatives of $S_{0}$ are really needed, e.g. $\frac{\delta S_{0}}{\delta \phi^{*}(x)}=-\left(\square+m^{2}\right) \phi(x)$, and the latter are well-defined functionals.
} 
For scalar QED and the symmetry given by the above defined $Q$, the MWI takes the particular form (cf. [6, Exer. 4.2.6])

$$
\begin{aligned}
\partial_{y}^{\mu} & T_{n+1}\left(B_{1}\left(x_{1}\right) \otimes \cdots \otimes B_{n}\left(x_{n}\right) \otimes j_{\mu}(y)\right)_{0} \\
= & \sum_{l=1}^{n} \delta\left(y-x_{l}\right) T_{n}\left(B_{1}\left(x_{1}\right) \otimes \cdots \otimes\left(\theta B_{l}\right)\left(x_{l}\right) \otimes \cdots \otimes B_{n}\left(x_{n}\right)\right)_{0} \\
& \quad-\partial_{y}^{\mu}\left(\sum_{l=1}^{n} \delta\left(y-x_{l}\right) T_{n}\left(B_{1}\left(x_{1}\right) \otimes \cdots \otimes\left(\theta_{\mu} B_{l}\right)\left(x_{l}\right) \otimes \cdots \otimes B_{n}\left(x_{n}\right)\right)_{0}\right)
\end{aligned}
$$

for $B_{1}, \ldots, B_{n} \in \mathcal{P}$, by using the AWI. Compared with (2.2), the additional terms (i.e. the terms in the last line) arise from the last term in the formula (3.6) for $\delta_{Q(y)} B(x)$.

Instead of the identities (2.4), we now obtain

$$
\begin{aligned}
& \partial_{\mu}^{y} T_{2}\left(\partial^{\nu} \phi(x) \otimes j^{\mu}(y)\right)_{0}=\delta(y-x) \partial^{\nu} \phi(x)_{0}-\left(\partial^{\nu} \delta\right)(y-x) \phi(x)_{0}, \\
& \partial_{\mu}^{y} T_{2}\left(\partial^{\nu} \phi^{*}(x) \otimes j^{\mu}(y)\right)_{0}=-\delta(y-x) \partial^{\nu} \phi^{*}(x)_{0}+\left(\partial^{\nu} \delta\right)(y-x) \phi^{*}(x)_{0}, \\
& \partial_{\mu}^{y} T_{2}\left(j^{\nu}(x) \otimes j^{\mu}(y)\right)_{0}=2 i\left(\phi^{*} \phi\right)(x)_{0} \partial^{\nu} \delta(y-x),
\end{aligned}
$$

by using $\theta^{\mu} j^{\nu}=-2 i g^{\mu \nu} \phi \phi^{*}$.

When working with the time-ordered product $T$ satisfying the MWI (3.7) one has to add the quartic interaction part 'by hand', that is, one starts the inductive Epstein-Glaser construction of the $S$-matrix with the following interaction $S$ :

$$
T_{1}(S)=S:=e\left(j^{\mu} A_{\mu}\right)(g)+e^{2}\left(A^{\mu} A_{\mu} \phi^{*} \phi\right)\left(g^{2}\right) \in \mathcal{F}_{\mathrm{loc}} .
$$

The addition of the quartic interaction term can be motivated by classical gauge invariance. In this procedure, the order of the time-ordered product does not agree with the order in the coupling constant $(e g)$; gauge invariance of the $S$-matrix must hold in each order in $(e g)$ individually.

Remark 3.1. [Off-shell MWI] The off-shell MWI differs from the on-shell MWI by additional terms proportional to the field equation for $\phi$ and $\phi^{*}$. In detail, the off-shell MWI for scalar QED is obtained from the on-shell MWI (3.7) by omitting the restriction of all $T$-products to $\mathcal{C}_{S_{0}}$ and by adding on the r.h.s. the two terms

$$
\begin{aligned}
& +i T_{n+1}\left(B_{1}\left(x_{1}\right) \otimes \cdots \otimes B_{n}\left(x_{n}\right) \otimes \phi(y)\right) \cdot\left(\square+m^{2}\right) \phi^{*}(y) \\
& -i T_{n+1}\left(B_{1}\left(x_{1}\right) \otimes \cdots \otimes B_{n}\left(x_{n}\right) \otimes \phi^{*}(y)\right) \cdot\left(\square+m^{2}\right) \phi(y) .
\end{aligned}
$$

The proof of the on-shell MWI given in Sect. 5 can be extended to the off-shell MWI by very minor supplements, as one sees by comparing with the proof of the off-shell MWI for spinor QED given in [6, Sect. 5.2.2]. 


\section{Equivalent Reformulations of the MWI}

Some remarks on the notations: in this section, we solely work with the timeordered product $T \equiv\left(T_{n}\right)$, which satisfies the AWI. Thus we may interpret $T_{n}$ as a map $T_{n}: \mathcal{F}_{\text {loc }}^{\otimes_{\mathrm{s}} n} \rightarrow \mathcal{F}$, and for the $S$-matrix (A.4), we may write

$$
\mathbf{S}(F) \equiv T\left(e_{\otimes_{\mathrm{s}}}^{i F}\right):=1+\sum_{n=1}^{\infty} \frac{i^{n}}{n !} T_{n}\left(F^{\otimes_{\mathrm{s}} n}\right)
$$

in the sense of formal power series in $F$, where $\otimes_{\mathrm{s}}$ denotes the symmetrized tensor product. In addition, let $B \in \mathcal{P}$ and $g, \alpha \in \mathcal{D}(\mathbb{M}, \mathbb{R})$ the function switching the coupling constant and an infinitesimal local $U(1)$-transformation, respectively.

\subsection{The MWI as an Identity for Formal Power Series}

Motivated by the expressions

$$
\begin{aligned}
& \int d y d x \alpha(y) g(x) \delta(y-x)(\theta B)(x)=(\theta B)(g \alpha), \\
& -\int d y d x \alpha(y) g(x) \partial_{y}^{\mu} \delta(y-x)\left(\theta_{\mu} B\right)(x)=\left(\theta_{\mu} B\right)\left(g \partial^{\mu} \alpha\right),
\end{aligned}
$$

which appear in the MWI (3.7) when integrated out with $\alpha(y) \prod_{j} g_{j}\left(x_{j}\right) \in$ $\mathcal{D}\left(\mathbb{M}^{n+1}, \mathbb{R}\right)$, we introduce two derivations (i.e. linear maps satisfying the Leibniz rule) on $\mathcal{T}\left(\mathcal{F}_{\text {loc }}\right)$ (by which we mean the the linear space spanned by the sequences $\left(F_{k, 1} \otimes_{\mathrm{s}} \cdots \otimes_{\mathrm{s}} F_{k, k}\right)_{k=0}^{\infty}$ in the tensor algebra on $\mathcal{F}_{\text {loc }}$, formally written as $\left.\sum_{k=0}^{\infty}\left(F_{k, 1} \otimes_{\mathrm{s}} \cdots \otimes_{\mathrm{s}} F_{k, k}\right)\right)$ :

$\delta_{\theta}^{(0)}(\alpha), \delta_{\theta}^{(1)}(\alpha): \mathcal{T}\left(\mathcal{F}_{\text {loc }}\right) \longrightarrow \mathcal{T}\left(\mathcal{F}_{\text {loc }}\right) \quad$ uniquely specified by $\delta_{\theta}^{(0)}(\alpha)(B(g)):=(\theta B)(g \alpha)$ and $\delta_{\theta}^{(1)}(\alpha)(B(g)):=\left(\theta_{\mu} B\right)\left(g \partial^{\mu} \alpha\right)$, respectively.

An immediate consequence is the relation

$$
d\left(e_{\otimes_{\mathrm{s}}}^{i F}\right)=i d(F) \otimes_{\mathrm{s}} e_{\otimes_{\mathrm{s}}}^{i F} \quad \text { for both } \quad d:=\delta_{\theta}^{(0)}(\alpha) \quad \text { and } \quad d:=\delta_{\theta}^{(1)}(\alpha) .
$$

In addition, looking at (3.4)-(3.6), we see that

$$
\delta_{\alpha Q}:=\int d y \alpha(y) \delta_{Q(y)}=-i\left(\delta_{\theta}^{(0)}(\alpha)+\delta_{\theta}^{(1)}(\alpha)\right) .
$$

With these tools we can give a more concise equivalent reformulation of the on-shell MWI (3.7):

$$
\begin{aligned}
T\left((\partial j)(\alpha) \otimes_{\mathrm{s}} e_{\otimes_{\mathrm{s}}}^{i F}\right)_{0}= & -T\left(\delta_{\alpha Q} F \otimes_{\mathrm{s}} e_{\otimes_{\mathrm{s}}}^{i F}\right)_{0} \\
\equiv & i T\left(\delta_{\theta}^{(0)}(\alpha)(F) \otimes_{\mathrm{s}} e_{\otimes_{\mathrm{s}}}^{i F}\right)_{0} \\
& +i T\left(\delta_{\theta}^{(1)}(\alpha)(F) \otimes_{\mathrm{s}} e_{\otimes_{\mathrm{s}}}^{i F}\right)_{0}, \quad \forall \alpha \in \mathcal{D}(\mathbb{M}, \mathbb{R}),
\end{aligned}
$$

which we understand as an identity for formal power series in $F \in \mathcal{F}_{\text {loc }}$. 
Conservation of the interacting current. As an application of the version (4.4) of the MWI, we study current conservation. For $S, G \in \mathcal{F}_{\text {loc }}$ let

$$
G_{S, 0}:=\mathbf{S}(S)_{0}^{\star-1} \star T\left(e_{\otimes_{\mathrm{s}}}^{i S} \otimes_{\mathrm{s}} G\right)_{0}
$$

be the interacting field to the interaction $S$ and corresponding to $G$, as defined by Bogoliubov [1]. More precisely, $G_{S, 0}$ is a formal power series in $S$ and to zeroth order in $S$ it agrees with $G_{0}$. For $S$ being the interaction of scalar QED (3.9), we obtain

$$
\delta_{\theta}^{(0)}(\alpha)(S)=0 \quad \text { and } \quad \delta_{\theta}^{(1)}(\alpha)(S)=-2 i e\left(\phi \phi^{*} A^{\mu}\right)\left(g \partial_{\mu} \alpha\right) .
$$

Now, in the MWI (4.4), we set $F:=S$ and multiply with $\mathbf{S}(S)_{0}^{\star-1} \star \cdots$. This yields

$$
-j(\partial \alpha)_{S, 0}=2 e\left(\phi \phi^{*} A\right)(g \partial \alpha)_{S, 0} .
$$

Omitting the arbitrary testfunction $\alpha$, this can be written as conservation of the interacting electromagnetic current:

$$
\begin{aligned}
& \partial_{\mu}^{x} J_{S}^{\mu}(x)_{0}=0 \quad \text { where } \\
& J^{\mu}(x):=j^{\mu}(x)+2 e g(x)\left(\phi \phi^{*} A\right)(x)=i\left(\phi(x)\left(D^{\mu} \phi\right)^{*}(x)-\phi^{*}(x) D^{\mu} \phi(x)\right)
\end{aligned}
$$

with the covariant derivative $D_{x}^{\mu}:=\partial_{x}^{\mu}+i e g(x) A^{\mu}(x) . J^{\mu}$ is the Noether current belonging to the invariance of the total action

$$
S_{0}+S=\int d x\left(\left(D^{\mu} \phi\right)^{*}(x) D^{\mu} \phi(x)-m^{2} \phi^{*}(x) \phi(x)\right)+S_{0}\left(A^{\mu}\right)
$$

(cf. (3.5)) under the same global $U(1)$-transformation $\phi(x) \rightarrow e^{i \alpha} \phi(x)$ as in the preceding sections. We recognize a further significant difference to spinor QED: the Noether currents $j$ and $J$ belonging to the free and interacting theory, respectively, are different.

\subsection{The Unitary MWI}

The Buchholz-Fredenhagen quantum algebra ('BF-algebra') [5] is an abstract $\mathrm{C}^{*}$-algebra (more precisely: a local net of $\mathrm{C}^{*}$-algebras) which, given the field content and a classical relativistic Lagrangian, encodes the pertinent interactions in QFT. In this generality, the most adequate formulation of symmetries is an open problem. A concrete algebra $\mathcal{A}$ fulfilling the defining relations of the BF-algebra belonging to the field content of scalar QED and the Lagrangian

$$
L_{0}:=\left(\partial \phi^{*} \partial \phi-m^{2} \phi^{*} \phi\right)-\frac{1}{4} F^{\mu \nu} F_{\mu \nu}
$$

(where $F^{\mu \nu}:=\partial^{\mu} A^{\nu}-\partial^{\nu} A^{\mu}$ ) is given by the perturbative on-shell $S$-matrices (4.1), that is, ${ }^{4}$

$$
\mathcal{A}:=\bigvee_{\star}\left\{\mathbf{S}(F)_{0} \mid F \in \mathcal{F}_{\text {loc }}\right\}
$$

\footnotetext{
${ }^{4} \mathrm{By}$ ' $\mathrm{V}_{\star}$ ' we mean the algebra, under the star product, generated by members of the indicated set. Also the analogous algebra generated by the off-shell $S$-matrices (i.e. without restriction to $\mathcal{C}_{S_{0}}$ ) fits into the definition of the $\mathrm{BF}$-algebra for the same field content and the same Lagrangian $L_{0}$; however, in view of the MWI, we prefer in the following to work on-shell.
} 
For this algebra, the above-mentioned problem amounts to the task of finding an equivalent reformulation of the MWI in terms of the maps $\mathcal{F}_{\text {loc }} \ni F \rightarrow$ $\mathbf{S}(F)_{0}$; in contrast to (4.4), expressions of the type $T\left(G \otimes_{\mathrm{s}} e_{\otimes_{\mathrm{s}}}^{i F}\right)_{0}$ must not appear.

For scalar QED, Fredenhagen has noted that the following conjectured identity [4] (see also [15]) would serve the purpose, which has some analogy to the Schwinger-Dyson equation: let

$$
\phi_{\alpha}(x):=\phi(x) e^{i \alpha(x)}, \quad \phi_{\alpha}^{*}(x):=\phi^{*}(x) e^{-i \alpha(x)}, \quad F_{\alpha}:=F\left(\phi_{\alpha}, \phi_{\alpha}^{*}, A^{\mu}\right),
$$

where $\alpha \in \mathcal{D}(\mathbb{M}, \mathbb{R})$ (see Remark 4.2 below) and define

$$
\delta L_{0}(\alpha):=\int d x\left(L_{0}(x)_{\alpha}-L_{0}(x)\right) .
$$

On the r.h.s. the range of integration is only $\operatorname{supp} \alpha$, that is, a bounded region. The conjecture asserts that the time-ordered product can be renormalized such that

$$
\mathbf{S}\left(F_{\alpha}+\delta L_{0}(\alpha)\right)_{0}=\mathbf{S}(F)_{0}, \quad \forall F \in \mathcal{F}_{\text {loc }}, \alpha \in \mathcal{D}(\mathbb{M}, \mathbb{R}) .
$$

We understand (4.9) as identity for formal power series in $\hbar, F$ and $\alpha,{ }^{5}$ and we will call it the 'unitary MWI' because it expresses the MWI in an equivalent way (as we show below) in terms of the $S$-matrix.

Setting $F:=0$ the unitary MWI reduces to

$$
\mathbf{S}\left(\delta L_{0}(\alpha)\right)_{0}=1 .
$$

For illustration, we explicitly compute $\delta L_{0}(\alpha)$. Taking into account that

$$
\partial_{x} \phi_{\alpha}(x)=(\partial \phi)(x) e^{i \alpha(x)}+i \phi_{\alpha}(x) \partial \alpha(x)
$$

and the analogous relation for $\partial_{x} \phi_{\alpha}^{*}(x)$, we obtain

$$
\delta L_{0}(\alpha)=-(\partial j)(\alpha)+\left(\phi^{*} \phi\right)\left((\partial \alpha)^{2}\right) .
$$

The following Theorem supports the conjecture:

Theorem 4.1. The unitary MWI (4.9) is equivalent to the on-shell MWI (4.4), when the latter is interpreted as an identity which should hold for all $F \in \mathcal{F}_{\text {loc }}$ and all $\alpha \in \mathcal{D}(\mathbb{M}, \mathbb{R})$.

Remark 4.2. Before giving the proof, we point out that $\alpha$ in (4.7) having compact support does not mean that the transformation underlying the unitary MWI is a local gauge transformation. Specifically, $A^{\mu}$ is not transformed. The test function $\alpha$ is used to control the dependence of functionals on the scalar field only, and its localization means that the transformation acts non-trivially only in a bounded region. Indeed, the theorem does not hold true for local gauge transformations, in particular the relation (4.13) becomes wrong, because then the l.h.s. of (4.13) contains additional terms coming from the transformation of $A^{\mu}$, but the r.h.s. does not contain such terms. A second reason becomes apparent by looking at the model containing only the electromagnetic field and assuming that $\alpha$ is a local gauge transformation. Then, it

\footnotetext{
${ }^{5}$ The dependence on $\hbar$ is not visible in our notations since we have set $\hbar:=1$, to simplify the notations.
} 
holds that $\delta L_{0}(\alpha)=0$. Hence, the conjectured formula (4.9) would be trivial for all observables $F$ (i.e. $F_{\alpha}=F$ ), hence worthless.

Proof. Let $0 \neq \beta \in \mathcal{D}(\mathbb{M}, \mathbb{R})$ be arbitrary and let $\alpha(x):=a \beta(x)$ with $a \in \mathbb{R}$. To prove that the MWI (4.4) implies the unitary MWI (4.9), let $\beta$ be fixed and interpret the l.h.s. of (4.9) as a function $f(a)$ of $a \in \mathbb{R}$, explicitly

$$
f(a):=T\left(e_{\otimes_{\mathrm{s}}}^{i G(a)}\right)_{0}, \quad \text { with } \quad G(a):=F_{a \beta}+\delta L_{0}(a \beta) .
$$

Since this function is differentiable (as we see from the explicit formulas) and since the unitary MWI holds trivially true for $a=0$, it suffices to show that $\frac{d}{d a} f(a)=0$ for all $a \in \mathbb{R}$ - as a consequence of the MWI (4.4). ${ }^{6}$

To prove $\frac{d}{d a} f(a)=0$, we use the following crucial relation for $F_{a \beta}$ :

$$
\frac{d}{d a} F_{a \beta}=-\delta_{\beta Q} F_{a \beta} \quad \forall F \in \mathcal{F}_{\text {loc }}
$$

Proof of (4.13): we compute the l.h.s. by using $\frac{d}{d a} \phi_{\alpha}(x)=i \beta(x) \phi_{\alpha}(x)$ :

$$
\begin{aligned}
\frac{d}{d a} F_{a \beta} & =\int d y\left(\frac{d \phi_{\alpha}(y)}{d a} \frac{\delta F_{a \beta}}{\delta \phi_{\alpha}(y)}+\frac{d \phi_{\alpha}^{*}(y)}{d a} \frac{\delta F_{a \beta}}{\delta \phi_{\alpha}^{*}(y)}\right) \\
& =i \int d y \beta(y)\left(\phi_{\alpha}(y) \frac{\delta F_{a \beta}}{\delta \phi_{\alpha}(y)}-\phi_{\alpha}^{*}(y) \frac{\delta F_{a \beta}}{\delta \phi_{\alpha}^{*}(y)}\right) .
\end{aligned}
$$

Taking into account that $\frac{\delta \phi_{\alpha}(z)}{\delta \phi(y)}=\delta(z-y) e^{i \alpha(z)}$, which implies

$$
\phi(y) \frac{\delta}{\delta \phi(y)}=\int d z \phi(y) \frac{\delta \phi_{\alpha}(z)}{\delta \phi(y)} \frac{\delta}{\delta \phi_{\alpha}(z)}=\phi_{\alpha}(y) \frac{\delta}{\delta \phi_{\alpha}(y)},
$$

and inserting $Q(y)=-i \phi(y)$, we obtain the assertion (4.13):

$$
\frac{d}{d a} F_{a \beta}=i \int d y \beta(y)\left(\phi(y) \frac{\delta F_{a \beta}}{\delta \phi(y)}-\phi^{*}(y) \frac{\delta F_{a \beta}}{\delta \phi^{*}(y)}\right)=-\delta_{\beta Q} F_{a \beta} .
$$

This concludes the proof of (4.13).

Now let $f \in \mathcal{D}(\mathbb{M}, \mathbb{R})$ with $\left.f\right|_{\operatorname{supp} \beta}=1$. Thanks to this property of $f$, it holds that

$$
\delta_{\beta Q} L_{0}(f)=\delta_{\beta Q} S_{0} \quad \text { and } \quad L_{0}(f)_{a \beta}-L_{0}(f) \stackrel{(4.8)}{=} \delta L_{0}(a \beta)
$$

where $L_{0}(f)_{a \beta}$ as a function of $a$ is defined similarly to $F_{a \beta}=F_{\alpha}$, i.e. $\alpha(x)=$ $a \beta(x)$. Note in particular, that for both equations in (4.15) also the 1.h.s.'s do not depend on the choice of $f$.

By applying the relation (4.13) to $L_{0}(f)_{a \beta}$, we obtain

$$
\begin{gathered}
\frac{d \delta L_{0}(a \beta)}{d a} \stackrel{(4.15)}{=} \frac{d L_{0}(f)_{a \beta}}{d a} \stackrel{(4.13)}{=}-\delta_{\beta Q} L_{0}(f)_{a \beta} \stackrel{(4.15)}{=}-\delta_{\beta Q}\left(\delta L_{0}(a \beta)\right)-\delta_{\beta Q}\left(L_{0}(f)\right) \\
\stackrel{(4.15)}{=}-\delta_{\beta Q}\left(\delta L_{0}(a \beta)\right)-\delta_{\beta Q} S_{0} .
\end{gathered}
$$

\footnotetext{
${ }^{6}$ For this function $f$, differentiability and the application of the fundamental theorem of calculus are understood in the sense of formal power series and functionals, that is, for each term of the formal series and applied to any fixed classical configuration.
} 
Equipped with these tools we are able to verify the vanishing of $\frac{d}{d a} f(a)$ for all $a \in \mathbb{R}$. The derivative can easily be computed:

$$
\begin{aligned}
\frac{d}{d a} f(a) & =i T\left(e_{\otimes_{\mathrm{s}}}^{i G(a)} \otimes_{\mathrm{s}}\left[\frac{d F_{a \beta}}{d a}+\frac{d \delta L_{0}(a \beta)}{d a}\right]\right)_{0} \\
& \stackrel{(4.13),(4.16)}{=}-i T\left(e_{\otimes_{\mathrm{s}}}^{i G(a)} \otimes_{\mathrm{s}}\left[\delta_{\beta Q} F_{a \beta}+\delta_{\beta Q}\left(\delta L_{0}(a \beta)\right)+\delta_{\beta Q} S_{0}\right]\right)_{0} \\
& =-i T\left(e_{\otimes_{\mathrm{s}}}^{i G(a)} \otimes_{\mathrm{s}}\left[\delta_{\beta Q} G(a)+\delta_{\beta Q} S_{0}\right]\right)_{0} ;
\end{aligned}
$$

the r.h.s. vanishes due to the MWI (4.4) for $G(a) \in \mathcal{F}_{\text {loc }}$, by remembering that $\delta_{\beta Q} S_{0}=\partial j(\beta)(3.6)$.

That the unitary MWI implies the MWI is obvious from our procedure: the former yields $\left.\frac{d}{d a}\right|_{a=0} T\left(e_{\otimes_{\mathrm{s}}}^{i G(a)}\right)_{0}=0$; after use of (4.17) this is the MWI (4.4) for $G(0)=F$ and $\beta$, which is the MWI in its full generality, because $F \in \mathcal{F}_{\text {loc }}$ and $\beta \in \mathcal{D}(\mathbb{M}, \mathbb{R})$ are arbitrary.

The fact that in this proof model-specific information is used only in the verification of the relation (4.13), indicates that the conjecture is valid also for other models with other symmetry transformations of the basic fields - see [4].

\section{Proof of the Master Ward Identity}

In this section, we prove the first main result of this paper, to wit, that the MWI (3.7) can be satisfied by a finite renormalization of the $T$-product for all $B_{1}, \ldots, B_{n} \in \mathcal{P}_{0}$, the set of

$L:=e j A+g e^{2} A^{2} \phi^{*} \phi, \quad j^{\mu}$ and all submonomials of these two field polynomials, except $e j A$ and $g e^{2} A^{2} \phi^{*} \phi$ and the individual parts of $j^{\mu}$ (i.e. $i \phi \partial^{\mu} \phi^{*}$ and $-i \phi^{*} \partial^{\mu} \phi$ ) separately. These exceptions are made to shorten the proof ${ }^{7}$; they are justified by the fact that physically relevant are only $L$ and $j^{\mu}$, that is, only the sums of their individual parts. The validity of the MWI (3.7) for the set $\mathcal{P}_{0}$ is sufficient for conservation of the interacting current (4.6) and (most probably) for a local construction of the main observables of scalar QED (including the interacting fields $\phi$ and $\phi^{*}$ ), in analogy to the construction given in [8] or [6, Chap. 5].

Note here a technical artifice: in the above definition of $L$ we have multiplied $e^{2}$ with $g(x)$, where $g \in \mathcal{D}(\mathbb{M})$ is arbitrary, in order that $L(g)$ agrees with the interaction $S$ given in (3.9). ${ }^{8}$ However, in all other elements of $\mathcal{P}_{0}$ (in particular, the proper submonomials of $g e^{2} A^{2} \phi^{*} \phi$ ), we do not include this factor $g$. Note that all $B_{j} \in \mathcal{P}_{0}$ are eigenvectors of $\theta$; we will use the notation $b_{j} B_{j}:=\theta B_{j}$.

We proceed in analogy with the proof of the QED-MWI in [6, Chap. 5.2.2], which relies on [8, App. B], and in addition we use specific arguments for scalar

\footnotetext{
${ }^{7}$ There is no reason to believe that the MWI (3.7) holds not true for a larger version of $\mathcal{P}_{0}$ containing these exceptions.

${ }^{8}$ Hence, as long as we do not perform the adiabatic limit $g(x) \rightarrow 1$, we investigate a theory with a spacetime dependent coupling constant $e g(x)$.
} 
QED given in [13]. Starting with a $T$-product fulfilling all other renormalization conditions (including the AWI and the Field Equation) and proceeding by induction on $n$, the anomalous term (i.e. the possible violation of the MWI) is given by

$$
\begin{aligned}
(-i)^{n} & \Delta^{n}\left(B_{1}\left(x_{1}\right), \ldots, B_{n}\left(x_{n}\right) ; y\right)_{0} \\
:= & -\partial_{y}^{\mu} T_{n+1}\left(B_{1}\left(x_{1}\right) \otimes \cdots \otimes B_{n}\left(x_{n}\right) \otimes j_{\mu}(y)\right)_{0} \\
& +\sum_{l=1}^{n} \delta\left(y-x_{l}\right) T_{n}\left(B_{1}\left(x_{1}\right) \otimes \cdots \otimes\left(\theta B_{l}\right)\left(x_{l}\right) \otimes \cdots \otimes B_{n}\left(x_{n}\right)\right)_{0} \\
& -\partial_{y}^{\mu}\left(\sum_{l=1}^{n} \delta\left(y-x_{l}\right) T_{n}\left(B_{1}\left(x_{1}\right) \otimes \cdots \otimes\left(\theta_{\mu} B_{l}\right)\left(x_{l}\right) \otimes \cdots \otimes B_{n}\left(x_{n}\right)\right)_{0}\right) .
\end{aligned}
$$

By using causal factorization of the $T$-products and the validity of the MWI to lower orders, one proves that

$$
\operatorname{supp} \Delta^{n}\left(B_{1}\left(x_{1}\right), \ldots, B_{n}\left(x_{n}\right) ; y\right)_{0} \subseteq \Delta_{n+1},
$$

where $\Delta_{n+1}$ denotes the thin diagonal in $\mathbb{M}^{n+1}$ (A.6), for details see $[6$, Chap. 4.2.2]. Therefore, the MWI (3.7) is indeed a renormalization condition, to be imposed on the definition of $T_{n+1}$ in the next perturbative order. The task is to remove $\Delta^{n}\left(B_{1}\left(x_{1}\right), \ldots ; y\right)_{0}$ by a finite, admissible renormalization of $T_{n+1}\left(B_{1}\left(x_{1}\right) \otimes \cdots \otimes B_{n}\left(x_{n}\right) \otimes j_{\mu}(y)\right)_{0}$, cf. (A.7). By 'admissible' we mean that the basic axioms and the above mentioned renormalization conditions (i)-(viii) and AWI and FE are maintained.

The idea of proof goes as follows: in the first two steps we prove $\int d y$ $\Delta^{n}(\cdots ; y)_{0}=0$. Because $\Delta^{n}(\cdots ; y)_{0}$ satisfies a version of the causal Wick expansion, it suffices to study the vacuum expectation value $d_{n}\left(B_{1}, \ldots\right)\left(x_{1}-\right.$ $y, \ldots):=\omega_{0}\left(\Delta^{n}\left(B_{1}\left(x_{1}\right), \ldots ; y\right)\right)$. By $(5.2), d_{n}\left(B_{1}, \ldots\right)\left(x_{1}-y, \ldots\right)$ is a linear combination of derivatives of $\delta\left(x_{1}-y, \ldots, x_{n}-y\right)$. By a version of the Poincaré Lemma, the result of the first two steps implies that $d_{n}\left(B_{1}, \ldots,\right)\left(x_{1}-y, \ldots\right)=$ $\partial_{\mu}^{y} u_{n}^{\mu}\left(B_{1}, \ldots\right)\left(x_{1}-y, \ldots\right)$ for some numerical distributions $u_{n}^{\mu}\left(B_{1}, \ldots\right)\left(x_{1}-\right.$ $y, \ldots)$ supported also on the thin diagonal. Therefore, the finite renormalization $t_{n+1}\left(B_{1}, \ldots, j^{\mu}\right) \rightarrow t_{n+1}\left(B_{1}, \ldots, j^{\mu}\right)+(-i)^{n} u_{n}^{\mu}\left(B_{1}, \ldots\right)$ removes $\Delta^{n}(\ldots ; y)_{0}$. One has to verify that the other renormalization conditions are maintained. Only one instance poses a serious difficulty: if at least one of the $B_{j}$ 's is a current $j^{\nu}$, it is not clear that this finite renormalization maintains the invariance of $t_{n+1}\left(B_{1}, \ldots, j^{\mu}\right)$ under permutation of the $j$ 's. We solve this problem by proceeding case by case.

Step 1: Similarly to [6, Exer. 5.1.7] one shows that

$$
\left[Q^{\phi}, B(x)_{0}\right]_{\star}=(\theta B)(x)_{0}, \quad \text { with } \quad Q^{\phi}:=\int d \vec{y} j^{0}(t, \vec{y})_{0},
$$

where the time $t \in \mathbb{R}$ is arbitrary and $[\cdot, \cdot]_{\star}$ denotes the commutator w.r.t. the star product. The integral $Q^{\phi}$ in (5.3) is meant symbolically, but its commutator understood as the integral over $\left[j^{0}(t, \vec{y})_{0}, B(x)_{0}\right]_{\star}$ is well-defined by locality. 
As it becomes clear below in Step 2, a necessary condition for the asserted MWI (3.7) is charge number conservation, which is a generalization of the relation (5.3) to time-ordered products of order $n \geq 2$, explicitly:

$$
\begin{aligned}
{\left[Q^{\phi}\right.} & \left., T_{n}\left(B_{1}\left(x_{1}\right) \otimes \cdots \otimes B_{n}\left(x_{n}\right)\right)_{0}\right]_{\star} \\
& =\sum_{l=1}^{n} T_{n}\left(B_{1}\left(x_{1}\right) \otimes \cdots \otimes\left(\theta B_{l}\right)\left(x_{l}\right) \otimes \cdots \otimes B_{n}\left(x_{n}\right)\right)_{0} \\
& =T_{n}\left(B_{1}\left(x_{1}\right) \otimes \cdots \otimes B_{n}\left(x_{n}\right)\right)_{0} \cdot \sum_{l=1}^{n} b_{l} .
\end{aligned}
$$

To explain how one can satisfy this relation, we first study a necessary condition for it, which is obtained by taking the VEV of $(5.4)$ : using $\omega_{0}\left(\left[Q^{\phi}, F\right]_{\star}\right)=$ 0 for any $F \in \mathcal{F}$ we get

$$
t_{n}\left(B_{1}, \ldots, B_{n}\right)=0 \quad \text { if } \quad \sum_{l=1}^{n} b_{l} \neq 0
$$

In the inductive construction of the $T$-products, the property (5.5) can get lost only in the extension to the thin diagonal, i.e. (5.5) is a renormalization condition. To fulfil it, we simply extend zero by zero - this is compatible with all other renormalization conditions. That (5.5) is also sufficient for (5.4) can be verified by means of the causal Wick expansion — for details see [6, Chap. 5.2.2]. So we assume in the following steps, that the $T$-products satisfy also (5.4). Note that we work here with the charge number operator $\theta$ only; $\theta_{\mu}$ does not play any role here.

Step 2: In this step we prove that charge number conservation (5.4) is equivalent to the relation

$$
\int d y \Delta^{n}\left(B_{1}\left(x_{1}\right), \ldots, B_{n}\left(x_{n}\right) ; y\right)_{0}=0
$$

Here, we understand the l.h.s. as a distribution in $\left(x_{1}, \ldots, x_{n}\right)$, i.e. smeared out with an arbitrary $h\left(x_{1}, \ldots, x_{n}\right) \in \mathcal{D}\left(\mathbb{M}^{n}\right)$. Thanks to this and (5.2), the integral is well-defined.

For a given configuration $\left(x_{1}, \ldots, x_{n}\right) \in \mathbb{M}^{n}$ let $\mathcal{O} \subset \mathbb{M}$ be an open double cone (i.e. the nonempty intersection of an open forward lightcone with an open backward lightcone) with $x_{1}, \ldots, x_{n} \in \mathcal{O}$; in addition let $f$ be an arbitrary test 
function satifying $\left.f\right|_{\overline{\mathcal{O}}}=1$. Thanks to $(5.2)$, we may write

$$
\begin{aligned}
(-i)^{n} & \int d y \Delta^{n}\left(B_{1}\left(x_{1}\right), \ldots, B_{n}\left(x_{n}\right) ; y\right)_{0} \\
= & (-i)^{n} \int d y f(y) \Delta^{n}\left(B_{1}\left(x_{1}\right), \ldots, B_{n}\left(x_{n}\right) ; y\right)_{0} \\
= & -\int d y f(y) \partial_{y}^{\mu} T\left(B_{1}\left(x_{1}\right) \otimes \cdots \otimes B_{n}\left(x_{n}\right) \otimes j_{\mu}(y)\right)_{0} \\
& +\sum_{l=1}^{n} T\left(B_{1}\left(x_{1}\right) \otimes \cdots \otimes\left(\theta B_{l}\right)\left(x_{l}\right) \otimes \cdots \otimes B_{n}\left(x_{n}\right)\right)_{0} \\
& +\sum_{l=1}^{n} \partial^{\mu} f\left(x_{l}\right) T\left(B_{1}\left(x_{1}\right) \otimes \cdots \otimes\left(\theta_{\mu} B_{l}\right)\left(x_{l}\right) \otimes \cdots \otimes B_{n}\left(x_{n}\right)\right)_{0},
\end{aligned}
$$

where we have integrated out the $\delta$-distributions. Compared with [6, eqn. (5.2.20)], there is an additional term appearing in the last line. However, because $\left.f\right|_{\overline{\mathcal{O}}}=$ 1 , this term vanishes. So we may continue as in that reference: we decompose $\partial^{\mu} f=a^{\mu}-b^{\mu}$ such that $\operatorname{supp} a^{\mu} \cap\left(\mathcal{O}+\bar{V}_{-}\right)=\emptyset$ and $\operatorname{supp} b^{\mu} \cap\left(\mathcal{O}+\bar{V}_{+}\right)=\emptyset$. By causal factorization of the $T$-products, the first term on the r.h.s. of (5.7) becomes

$$
\begin{aligned}
& j^{\mu}\left(a_{\mu}\right)_{0} \star T\left(B_{1}\left(x_{1}\right) \otimes \cdots \otimes B_{n}\left(x_{n}\right)\right)_{0}-T\left(B_{1}\left(x_{1}\right) \otimes \cdots \otimes B_{n}\left(x_{n}\right)\right)_{0} \star j^{\mu}\left(b_{\mu}\right)_{0} \\
& =\left[j^{\mu}\left(a_{\mu}\right)_{0}, T\left(B_{1}\left(x_{1}\right) \otimes \cdots \otimes B_{n}\left(x_{n}\right)\right)_{0}\right]_{\star} \\
& \quad+T\left(B_{1}\left(x_{1}\right) \otimes \cdots \otimes B_{n}\left(x_{n}\right)\right)_{0} \star j^{\mu}\left(\partial_{\mu} f\right)_{0} .
\end{aligned}
$$

The second term vanishes because $\partial_{\mu} j_{0}^{\mu}=0$. From the Field Independence of $T$ we know that $\operatorname{supp} T\left(\widetilde{B}_{1}\left(x_{1}\right) \otimes \cdots \otimes \widetilde{B}_{n}\left(x_{n}\right)\right)_{0} \subset \mathcal{O}$; therefore, we may vary $a^{\mu}$ in the set

$\left\{z \in \mathbb{M} \mid(z-x)^{2}<0 \forall x \in \mathcal{O}\right\} \quad$ without affecting $\quad\left[j^{\mu}\left(a_{\mu}\right)_{0}, T\left(\widetilde{B}_{1}\left(x_{1}\right) \otimes \cdots\right)_{0}\right]_{\star}$.

In particular we may choose for $a_{\mu}$ a smooth approximation to $\partial_{\mu} \theta\left(c-x^{0}\right)=$ $-\delta_{\mu 0} \delta\left(x^{0}-c\right)$, where $c \in \mathbb{R}$ is a sufficiently large constant:

$$
a_{\mu}(x)=-\delta_{\mu 0} h\left(x^{0}\right) \quad \text { with } \quad \int d x^{0} h\left(x^{0}\right)=1, \quad h \in \mathcal{D}([c-\varepsilon, c+\varepsilon])
$$

for some $\varepsilon>0$. Then, we obtain

$$
\begin{aligned}
& {\left[j^{\mu}\left(a_{\mu}\right)_{0}, T(\ldots)_{0}\right]_{\star}=-\int d x^{0} h\left(x^{0}\right) \int d \vec{x}\left[j^{0}\left(x^{0}, \vec{x}\right)_{0}, T(\ldots)_{0}\right]_{\star}} \\
& =-\int d \vec{x}\left[j^{0}(c, \vec{x})_{0}, T(\ldots)_{0}\right]_{\star}\left(\int d x^{0} h\left(x^{0}\right)\right)=-\left[Q^{\phi}, T(\ldots)_{0}\right]_{\star} .
\end{aligned}
$$

Inserting these results into (5.7), we see that charge number conservation (5.4) implies the assertion (5.6).

Step 3: Following the proof of the QED-MWI we list some structural properties of the anomalous term $\Delta^{n}(\cdots)$ defined in (5.1), for the validity of these properties see the above mentioned references. 
First, $\Delta^{n}(\cdots)$ satisfies the following version of the causal Wick expansion (cf. (A.2)):

$$
\begin{aligned}
\Delta^{n} & \left(B_{1}\left(x_{1}\right), \ldots, B_{n}\left(x_{n}\right) ; y\right) \\
& =\sum_{\underline{B}_{l} \subseteq B_{l}} d_{n}\left(\underline{B}_{1}, \ldots, \underline{B}_{n}\right)\left(x_{1}-y, \ldots\right) \bar{B}_{1}\left(x_{1}\right) \cdots \bar{B}_{n}\left(x_{n}\right),
\end{aligned}
$$

where the sum runs over all submonomials $\underline{B}_{l}$ of $B_{l}$ (where $1 \leq l \leq n$ ), and

$$
\begin{aligned}
& d_{n}\left(B_{1}, \ldots, B_{n}\right)\left(x_{1}-y, \ldots, x_{n}-y\right) \\
& \quad:=\omega_{0}\left(\Delta^{n}\left(B_{1}\left(x_{1}\right), \ldots, B_{n}\left(x_{n}\right) ; y\right)\right) \in \mathcal{D}^{\prime}\left(\mathbb{M}^{n}\right) .
\end{aligned}
$$

If $B_{j}=L$ and $\underline{B}_{j}$ is a proper submonomial of $g e^{2} A^{2} \phi^{*} \phi$, we include the factor $g\left(x_{j}\right)$ in $\bar{B}_{j}\left(x_{j}\right)$. This is consistent, since, due to $T\left(\cdots \otimes g\left(x_{j}\right)\left(A^{2} \phi^{*} \phi\right)\left(x_{j}\right) \otimes\right.$ $\cdots)=g\left(x_{j}\right) T\left(\cdots \otimes\left(A^{2} \phi^{*} \phi\right)\left(x_{j}\right) \otimes \cdots\right)$, it holds that $\Delta^{n}\left(\ldots, g\left(x_{j}\right)\left(A^{2} \phi^{*} \phi\right)\left(x_{j}\right)\right.$, $\ldots ; y)=g\left(x_{j}\right) \Delta^{n}\left(\ldots,\left(A^{2} \phi^{*} \phi\right)\left(x_{j}\right), \ldots ; y\right)$.

The validity of (5.8) can be traced back to the validity of the causal Wick expansion for the $T$-products appearing on the r.h.s. of (5.1); the fact that there is no term in (5.8) coming from proper submonomials of $\partial^{\mu} j_{\mu}$ is due to the validity of the axiom FE-for details see [6, Thm. 4.3.1 and Chap. 5.2.2].

If one of the $B_{j}$ 's is linear in the basic fields, e.g. $B_{1}=\partial^{a} \phi$ with $a \in \mathbb{N}^{4}$, the axiom FE determines uniquely $T_{n+1}\left(\partial^{a} \phi\left(x_{1}\right) \otimes \cdots \otimes j_{\mu}(y)\right)$ in terms of $T_{k}$ with $k \leq n$, that is, there is no freedom to remove $\Delta^{n}\left(\partial^{a} \phi\left(x_{1}\right) \otimes \cdots\right)$ by a finite renormalization of this $T$-product. However, as verified in [6, Exer. 4.3.3], the validity of the axiom $\mathrm{FE}$ implies that $\Delta^{n}(\cdots)$ vanishes in this case. Therefore, on the r.h.s. of (5.8), the sum is restricted to submonomials $\underline{B}_{l}$ of $B_{l}$ which are at least quadratic in the basic fields for all $l$.

From (5.2) we conclude that $d_{n}\left(B_{1}, \ldots, B_{n}\right)\left(x_{1}-y, \ldots, x_{n}-y\right)$ is a linear combination of derivatives of $\delta\left(x_{1}-y, \ldots, x_{n}-y\right)$. Using in addition a version of the Poincaré Lemma (more precisely, [6, Lemma 4.5.1]), the property

$$
\int d y d_{n}\left(B_{1}, \ldots, B_{n}\right)\left(x_{1}-y, \ldots, x_{n}-y\right)=0
$$

(which is obtained by taking the VEV of the corresponding relation for $\Delta^{n}$ (5.6)) implies that we can write $d\left(B_{1}, \ldots, B_{n}\right)$ as

$$
d_{n}\left(B_{1}, \ldots, B_{n}\right)\left(x_{1}-y, \ldots, x_{n}-y\right)=\partial_{\mu}^{y} u_{n}^{\mu}\left(B_{1}, \ldots, B_{n}\right)\left(x_{1}-y, \ldots, x_{n}-y\right)
$$

where $u_{n}^{\mu}\left(B_{1}, \ldots, B_{n}\right)$ is Lorentz covariant and of the form

$$
u_{n}^{\mu}\left(B_{1}, \ldots, B_{n}\right)\left(x_{1}-y, \ldots\right)=\sum_{a \in \mathbb{N}^{4 n}} C_{a}\left(B_{1}, \ldots, B_{n}\right) \partial^{a} \delta\left(x_{1}-y, \ldots\right) .
$$


Since $d_{n}\left(B_{1}, \ldots, B_{n}\right)$ is defined by the VEV of the r.h.s. of $(5.1),{ }^{9}$

$$
\begin{aligned}
(-i)^{n} & d_{n}\left(B_{1}, \ldots, B_{n}\right)\left(x_{1}-y, \ldots, x_{n}-y\right) \\
:= & -\partial_{\mu}^{y} t_{n+1}\left(B_{1}, \ldots, B_{n}, j^{\mu}\right)\left(x_{1}-y, \ldots, x_{n}-y\right) \\
& +\sum_{l=1}^{n} b_{l} \delta\left(y-x_{l}\right) t_{n}\left(B_{1}, \ldots, B_{n}\right)\left(x_{1}-x_{n}, \ldots, x_{n-1}-x_{n}\right) \\
& -\partial_{\mu}^{y}\left(\sum_{l=1}^{n} \delta\left(y-x_{l}\right) t_{n}\left(B_{1}, \ldots,\left(\theta^{\mu} B_{l}\right), \ldots, B_{n}\right)\left(x_{1}-x_{n}, \ldots, x_{n-1}-x_{n}\right)\right),
\end{aligned}
$$

we obtain an upper bound for the scaling degree of $d_{n}\left(B_{1}, \ldots, B_{n}\right)$ by the maximum of the scaling degrees of the terms standing on the r.h.s. of (5.12). Proceeding this way, we see that the sum over $a$ in (5.11) is bounded by

$$
|a| \leq \omega\left(B_{1}, \ldots, B_{n}\right)-1 \quad \text { where } \omega\left(B_{1}, \ldots, B_{n}\right):=\sum_{j=1}^{n} \operatorname{dim} B_{j}+4-4 n .
$$

Step 4: Obviously, the finite renormalization

$$
t_{n+1}\left(B_{1}, \ldots, B_{n}, j^{\mu}\right) \rightarrow t_{n+1}\left(B_{1}, \ldots, B_{n}, j^{\mu}\right)+(-i)^{n} u_{n}^{\mu}\left(B_{1}, \ldots, B_{n}\right)
$$

removes the anomalous term $d_{n}\left(B_{1}, \ldots, B_{n}\right)$. Looking at the causal Wick expansion of $\Delta_{n}$ (5.8), we conclude: performing the finite renormalization (5.14) for all $B_{1}, \ldots, B_{n} \in \mathcal{P}_{0}$ being at least quadratic in the basic fields, the MWI (3.7) is proved for all $B_{1}, \ldots, B_{n} \in \mathcal{P}_{0}$, provided that all these finite renormalizations are admissible, that is, they maintain the basic axioms and the renormalization conditions (v)-(viii), AWI and FE.

This is obvious for ${ }^{10}$ (i) Linearity (the set $\mathcal{P}_{0}$ is linearly independent), (iv) Causality, (v) Field Independence, (vii) Poincaré Covariance and FE Field Equation (all $B_{j}$ 's in (5.14) are at least bilinear in the basic fields). The maintenance of the axiom (viii) Scaling Degree follows from (5.13).

Concerning the axiom (vi) $*$-Structure, first note that $d_{n}\left(B_{1}^{*}, \ldots, B_{n}^{*}\right)=$ $\overline{d_{n}\left(B_{1}, \ldots, B_{n}\right)}[6$, formula $(5.2 .26)]$. Hence, to preserve this axiom, we may replace $u_{n}\left(B_{1}, \ldots, B_{n}\right)$ by $\frac{1}{2}\left(u_{n}\left(B_{1}, \ldots, B_{n}\right)+\overline{u_{n}\left(B_{1}^{*}, \ldots, B_{n}^{*}\right)}\right)$.

To maintain the axiom AWI, we also perform a finite renormalization of $t_{n+1}\left(B_{1}, \ldots, \partial B_{j}, \ldots, j^{\mu}\right)$ and $\left(B_{1}, \ldots, B_{n}, \partial^{\nu} j^{\mu}\right)$ such that the renormalized $t_{n+1}$ satisfies the AWI: $\partial_{x_{j}} t_{n+1}\left(\ldots, B_{j}, \ldots\right)=t_{n+1}\left(\ldots, \partial B_{j}, \ldots\right)$. In particular, for $B_{j}=\phi \phi^{*}$ we may renormalize $t_{n+1}\left(\ldots, \partial B_{j}, \ldots\right)$ independently from the renormalization of $t_{n+1}(\ldots, j, \ldots)$, since $j=i\left(\phi \partial \phi^{*}-(\partial \phi) \phi^{*}\right)$. The validity of the AWI for $t_{n+1}$ implies the validity of the AWI for the pertinent $T_{n+1}$ constructed by the causal Wick expansion, as explained in [6, Chap. 3.2.4, Step 3]. (The wave front set condition poses no problem by translation invariance of $u_{n}^{\mu}$, cf. [6, Remark 1.2.6].)

\footnotetext{
${ }^{9}$ See (A.5) for the definition of $t_{n+1}$ and $t_{n}$, respectively.

${ }^{10}$ We use the numbering of the axioms given in Appendix A.1.
} 
Only the maintenance of the axiom (iii) Symmetry poses a serious difficulty: if at least one of the $B_{j}$ 's is a current $j^{\nu}$, it is not clear whether the finite renormalization (5.14) preserves the invariance of

$$
t_{n+1}\left(B_{1}, \ldots, B_{l}, j^{\nu_{1}}, \ldots, j^{\nu_{k}}, j^{\mu}\right)\left(x_{11}-y, \ldots, x_{1 l}-y, x_{21}-y, \ldots, x_{2 k}-y\right)
$$

(where $l+k=n$ ) under the permutations of the entries pertaining to the currents, that is, under $\left(x_{2 r}, \nu_{r}\right) \leftrightarrow(y, \mu)$ for all $1 \leq r \leq k$. Taking additionally into account that $u_{n}^{\mu}\left(B_{1}, \ldots, B_{n}\right)$ is not uniquely determined by $d_{n}\left(B_{1}, \ldots, B_{n}\right)$ (one may add to $u_{n}^{\mu}$ some $\widetilde{u}_{n}^{\mu}$ with $\partial_{\mu}^{y} \widetilde{u}_{n}^{\mu}=0$ ), the remaining task can be formulated as follows: for any $B_{1}, \ldots, B_{l} \in \mathcal{P}_{0} \backslash\left\{j^{\nu}\right\}$ being at least quadratic in the basic fields, with $l \leq n-1$, and satisfying

$$
1 \leq \omega\left(B_{1}, \ldots, B_{l}, j^{\nu_{1}}, \ldots, j^{\nu_{n-l}}\right)=\sum_{s=1}^{l} \operatorname{dim} B_{s}+4-n-3 l
$$

(where $\operatorname{dim} j^{\nu}=3$ is used), we have to find distributions $u_{n}^{\mu}\left(B_{1}, \ldots, B_{l}\right.$, $\left.j^{\nu_{1}}, \ldots, j^{\nu_{k}}\right)$ of the form (5.11) having the same permutation symmetries and the same Lorentz covariance properties as $t_{n+1}\left(B_{1}, \ldots, B_{l}, j^{\nu_{1}}, \ldots, j^{\nu_{k}}, j^{\mu}\right)$, and which fulfil the equation (5.10) for $d_{n}\left(B_{1}, \ldots, B_{l}, j^{\nu_{1}}, \ldots, j^{\nu_{k}}\right)$ given by (5.12).

Looking at (5.10) note that even if the distributions $d_{n}=\partial_{\mu}^{y} u_{n}^{\mu}$ are invariant under the action on coordinates and indices of a group $G$ of permutations $\pi$, and the operation $\partial_{\mu}^{y}$ commutes with this action, then $u_{n}$ does in general not share this symmetry; but we may redefine it such that it does. In detail, calling $p_{\pi}$ the relevant representation of $\pi$ and $p:=\frac{1}{|G|} \sum_{\pi \in G} p_{\pi}$ the symmetrizer, we may replace

$u_{n} \quad$ by $\quad p u_{n}, \quad$ because $\partial_{\mu}^{y}\left(p u_{n}^{\mu}\right)=p\left(\partial_{\mu}^{y} u_{n}^{\mu}\right)=p d_{n}=d_{n}$.

For some $n$-tuples $\left(B_{1}, \ldots, B_{l}, j^{\nu_{1}}, \ldots, j^{\nu_{n-l}}\right)$ satisfying (5.16), we know that $d_{n}\left(B_{1}, \ldots, B_{l}, j^{\nu_{1}}, \ldots, j^{\nu_{n-l}}\right)=0$ due to charge number conservation $(C N C)$ or Furry's theorem (FT). In detail:

CNC By using (5.5)

$$
\left(B_{1}, \ldots, B_{l}, j^{\nu_{1}}, \ldots, j^{\nu_{n-l}}\right) \quad \text { satisfying } \sum_{s=1}^{l} b_{s} \neq 0
$$

all distributions $t_{n+1}(\ldots)$ and $t_{n}(\ldots)$ appearing on the r.h.s. of (5.12) vanish, hence $d_{n}(\ldots)=0$.

FT Charge conjugation is a linear operator $\beta_{C}: \mathcal{F} \rightarrow \mathcal{F}$ which is given by the relations

$$
\begin{aligned}
& \beta_{C}\left(\partial^{a} \phi(x)\right)=\eta_{C} \partial^{a} \phi^{*}(x), \quad \beta_{C}\left(\partial^{a} \phi^{*}(x)\right)=\bar{\eta}_{C} \partial^{a} \phi(x) \\
& \text { and } \beta_{C}\left(\partial^{a} A^{\mu}(x)\right)=-\left(\partial^{a} A^{\mu}(x)\right),
\end{aligned}
$$

where $\eta_{C} \in\{z \in \mathbb{C}|| z \mid=1\}$ is a fixed number, and by

$$
\begin{aligned}
& \beta_{C}\left\langle f_{p, n, l}^{\mu_{1} \ldots},\left(\otimes_{i=1}^{p} A_{\mu_{i}}\right) \otimes \phi^{\otimes n} \otimes\left(\phi^{*}\right)^{\otimes l}\right\rangle \\
& \quad:=\left\langle f_{p, n, l}^{\mu_{1} \ldots},\left(\otimes_{i=1}^{p} \beta_{C} A_{\mu_{i}}\right) \otimes\left(\beta_{C} \phi\right)^{\otimes n} \otimes\left(\beta_{C} \phi^{*}\right)^{\otimes l}\right\rangle
\end{aligned}
$$


(where (1.3) is used); for details about charge conjugation in the starproduct formalism of this paper, see [6, Chap. 5.1.5].

Charge Conjugation Invariance is the condition

$$
\beta_{C} \circ T_{n}=T_{n} \circ \beta_{C}^{\otimes n}
$$

on the $T$-product. Analogously to spinor QED (see, e.g. [6, Chap. 5.1.5] again), one verifies that this is an additional renormalization condition, which can be fulfilled such that all other renormalization conditions are preserved.

Furry's theorem is a consequence of (5.18), obtained by using that $\omega_{0} \circ$ $\beta_{C}=\omega_{0}$. It states: Let $A_{i}, B_{j} \in \mathcal{P}, i=1, \ldots, r, j=1, \ldots, s$ with $\beta_{C} A_{i}=$ $A_{i}$ and $\beta_{C} B_{j}=-B_{j}$ for all $i, j$. Then it holds that

$$
t_{r+s}\left(A_{1}, \ldots, A_{r}, B_{1}, \ldots, B_{s}\right)=0 \text { if } s \text { is odd. }
$$

Looking at the definition of $d_{n}(\ldots)$ (5.12) for the $n$-tuples

$$
(L, \ldots, L, j, j), \quad\left(L, \ldots, L, A \phi^{*} \phi, j\right),
$$

we verify that all distributions $t_{n+1}(\ldots)$ and $t_{n}(\ldots)$ appearing on the r.h.s. vanish, due to $(5.19)$, hence $d_{n}(\ldots)=0$. For this verification, we also use the following relations:

$$
\begin{aligned}
& \beta_{C} L=L, \quad \beta_{C} j^{\mu}=-j^{\mu}, \quad \theta L=0, \quad \theta j=0, \quad \theta^{\mu} L=-2 i e A^{\mu} \phi^{*} \phi, \\
& \beta_{C}\left(A^{\mu} \phi^{*} \phi\right)=-A^{\mu} \phi^{*} \phi, \quad \beta_{C}\left(\theta^{\mu} j^{\nu}\right)=\theta^{\mu} j^{\nu}, \quad \theta\left(A \phi^{*} \phi\right)=0, \quad \theta^{\mu}\left(A^{\nu} \phi^{*} \phi\right)=0 .
\end{aligned}
$$

There remain the $n$-tuples listed in the following table, that we shall study case-by-case. In each case, the distribution $u_{n}^{\mu}(\ldots)$ for the finite renormalization in (5.14) stands for a Lorentz tensor of rank $\geq 2$ according to the entries $B_{1}, \ldots, B_{n}$. It is a multiple of the total $\delta$-distribution in all arguments in all cases except Case 1 , where the scaling degree admits two derivatives. Therefore, we begin with the simpler cases 3 and 2, before we turn to the more delicate case 1 . We use the labelling of the $x$ variables (i.e. of the arguments of $\left.B_{1}, \ldots, B_{n}\right)$ indicated in $(5.15)$.

Cases 3: By (5.10)-(5.13), the renormalization is a Lorentz tensor $u_{n}^{\mu \nu}$ of rank 2 . The only possibility is

$$
u_{n}^{\mu \nu}\left(x_{11}-y, \ldots, x_{2}-y\right)=C g^{\mu \nu} \delta\left(x_{11}-y, \ldots, x_{2}-y\right),
$$

for some $C \in \mathbb{C}$. Obviously, $u_{n}^{\mu \nu}\left(\ldots, x_{2}-y\right)$ is invariant under $\left(\nu, x_{2}\right) \leftrightarrow(\mu, y)$, hence, the finite renormalization (5.14) is admissible in this case.

Cases 2a,b,c: Here, $u_{n}^{\mu}$ is a Lorentz tensor of rank 4 which is a multiple of the $\delta$-distribution,

$$
\begin{aligned}
& u_{n}^{\mu \nu_{1} \nu_{2} \nu_{3}}\left(x_{11}-y, \ldots, x_{21}-y, \ldots, x_{23}-y\right) \\
& \quad=\left(C_{1} g^{\mu \nu_{1}} g^{\nu_{2} \nu_{3}}+C_{2} g^{\mu \nu_{2}} g^{\nu_{1} \nu_{3}}+C_{3} g^{\mu \nu_{3}} g^{\nu_{1} \nu_{2}}\right) \prod_{r=1}^{n-3} \delta\left(x_{1 r}-y\right) \cdot \prod_{s=1}^{3} \delta\left(x_{2 s}-y\right),
\end{aligned}
$$

for some $C_{k} \in \mathbb{C}$. The totally antisymmetric tensor $\epsilon^{\mu \nu_{1} \nu_{2} \nu_{3}}$ is ruled out because because we may redefine $u_{n}^{\mu \nu_{1} \nu_{2} \nu_{3}}$ such that it is symmetric under $\left(\nu_{s_{1}}, x_{2 s_{1}}\right) \leftrightarrow$ 


\begin{tabular}{lll}
\hline$B_{1}, \ldots, B_{n}$ & $\omega\left(B_{1}, \ldots, B_{n}\right)$ & case number \\
\hline$\underbrace{L, \ldots, L}_{n-1}, j^{\nu}$ & 3 & 1 \\
$\underbrace{L, \ldots, L}_{n-3}, j^{\nu_{1}}, j^{\nu_{2}}, j^{\nu_{3}}$ & 1 & $2 \mathrm{a}$ \\
$\underbrace{L, \ldots, L}_{n-3}, A^{\nu_{1}} \phi^{*} \phi, A^{\nu_{2}} \phi^{*} \phi, j^{\nu_{3}}$ & 1 & $2 \mathrm{~b}$ \\
$\underbrace{L, \ldots, L}_{n-3}, A^{\nu_{1}} \phi^{*} \phi, j^{\nu_{2}}, j^{\nu_{3}}$ & 1 & $2 \mathrm{c}$ \\
$\underbrace{L, \ldots, L}_{n-2}, \phi^{*} \phi, j^{\nu}$ & 1 & 3 \\
$\underbrace{L, \ldots, L}_{n-2}, A^{2}, j^{\nu}$ & 1 & 3 \\
$\underbrace{L, \ldots, L}_{n-3}, A^{2} \phi, A^{2} \phi^{*}, j^{\nu}$ & 1 & 3 \\
$\underbrace{L, \ldots, L}_{n-3}, A \partial \phi, A \partial \phi^{*}, j^{\nu}$ & 1 & 3 \\
$\underbrace{L, \ldots, L}_{n-3}, A^{2} \phi, A \partial \phi^{*}, j^{\nu}$ & 1 & 3
\end{tabular}

$\left(\nu_{s_{2}}, x_{2 s_{2}}\right)$ for at least one pair $\left(s_{1}, s_{2}\right)$, see (5.17). Similarly, up to a redefinition of $u_{n}$, we may assume that $u_{n}^{\mu \nu_{1} \nu_{2} \nu_{3}}$ shares the symmetry of $d_{n}^{\nu_{1} \nu_{2} \nu_{3}}\left(B_{1}, \ldots, j^{\nu_{3}}\right)$ under permutation(s) of the pairs

$$
\begin{cases}\left(\nu_{1}, x_{21}\right),\left(\nu_{2}, x_{22}\right),\left(\nu_{3}, x_{23}\right) & \text { in case } 2 \mathrm{a} \\ \left(\nu_{1}, x_{21}\right),\left(\nu_{2}, x_{22}\right) & \text { in case } 2 \mathrm{~b} ; \\ \left(\nu_{2}, x_{22}\right),\left(\nu_{3}, x_{23}\right) & \text { in case } 2 \mathrm{c}\end{cases}
$$

Consequently

$$
\begin{cases}C_{1}=C_{2}=C_{3} & \text { in case } 2 \mathrm{a} \\ C_{1}=C_{2} & \text { in case } 2 \mathrm{~b} . \\ C_{2}=C_{3} & \text { in case } 2 \mathrm{c}\end{cases}
$$

Hence, in cases $2 \mathrm{a}$ and $2 \mathrm{~b}$ we have accomplished that $u_{n}^{\mu \nu_{1} \nu_{2} \nu_{3}}$ has the needed permutation symmetries to be an admissible finite renormalization.

In the case $2 \mathrm{c}$, the symmetry of $u_{n}^{\mu \nu_{1} \nu_{2} \nu_{3}}$ under permutations of the three currents requires $C_{1}=C_{2}=C_{3}$, which is not already secured by (5.22). To complete the proof for case 2c, we claim that

$$
\partial_{\nu_{3}}^{x_{23}} d_{n}\left(L, \ldots, L, A^{\nu_{1}} \phi^{*} \phi, j^{\nu_{2}}, j^{\nu_{3}}\right)\left(x_{11}-y, \ldots, x_{21}-y, \ldots, x_{23}-y\right)
$$

is invariant under $x_{23} \leftrightarrow y$. To verify this claim, we insert the definition of $d_{n}$ (5.12). Up to a global prefactor $i^{n}$, we obtain for $\partial_{\nu_{3}}^{x_{23}} d_{n}$ the following sum of 
terms: ${ }^{11}$

$$
\begin{aligned}
& -\partial_{\nu_{3}}^{x_{23}} \partial_{\mu}^{y} t_{n+1}\left(L\left(x_{11}\right), \ldots,\left(A^{\nu_{1}} \phi^{*} \phi\right)\left(x_{21}\right), j^{\nu_{2}}\left(x_{22}\right), j^{\nu_{3}}\left(x_{23}\right), j^{\mu}(y)\right) \\
& -\sum_{l=1}^{n-3} \partial_{\mu}^{y} \delta\left(y-x_{1 l}\right) \partial_{\nu_{3}}^{x_{23}} t_{n}\left(L\left(x_{11}\right), \ldots,\left(\theta^{\mu} L\right)\left(x_{1 l}\right), \ldots,\right. \\
& \left.\quad\left(A^{\nu_{1}} \phi^{*} \phi\right)\left(x_{21}\right), j^{\nu_{2}}\left(x_{22}\right), j^{\nu_{3}}\left(x_{23}\right)\right) \\
& +2 i e \partial_{y}^{\nu_{2}} \delta\left(y-x_{22}\right) \partial_{\nu_{3}}^{x_{23}} t_{n}\left(L\left(x_{11}\right), \ldots,\left(A^{\nu_{1}} \phi^{*} \phi\right)\left(x_{21}\right),\left(\phi^{*} \phi\right)\left(x_{22}\right), j^{\nu_{3}}\left(x_{23}\right)\right) \\
& +2 i e \partial_{\mu}^{y} \partial_{x_{23}}^{\mu}\left(\delta\left(y-x_{23}\right) t_{n}\left(L\left(x_{11}\right), \ldots,\left(A^{\nu_{1}} \phi^{*} \phi\right)\left(x_{21}\right), j^{\nu_{2}}\left(x_{22}\right),\left(\phi^{*} \phi\right)\left(x_{23}\right)\right)\right) .
\end{aligned}
$$

Obviously, the terms (5.24) and (5.27) are individually invariant under $x_{23} \leftrightarrow$ $y$. To show this for the sum of the remaining terms, we insert the MWI to order $(n-1)$, which holds by induction:

$$
\begin{aligned}
{[(5.25)] } & =\sum_{l \neq k} \partial_{\mu}^{y} \delta\left(y-x_{1 l}\right) \partial_{\nu_{3}}^{x_{23}} \delta\left(x_{23}-x_{1 k}\right) \\
& \cdot t_{n-1}\left(\ldots,\left(\theta^{\mu} L\right)\left(x_{1 l}\right), \ldots,\left(\theta^{\nu_{3}} L\right)\left(x_{1 k}\right), \ldots,\left(A^{\nu_{1}} \phi^{*} \phi\right)\left(x_{21}\right), j^{\nu_{2}}\left(x_{22}\right)\right) \\
& -2 i e \sum_{l} \partial_{\mu}^{y} \delta\left(y-x_{1 l}\right) \partial_{x_{23}}^{\nu_{2}} \delta\left(x_{23}-x_{22}\right) \\
& \cdot t_{n-1}\left(\ldots,\left(\theta^{\mu} L\right)\left(x_{1 l}\right), \ldots,\left(A^{\nu_{1}} \phi^{*} \phi\right)\left(x_{21}\right),\left(\phi^{*} \phi\right)\left(x_{22}\right)\right) \\
{[(5.26)]=} & -2 i e \sum_{l} \partial_{y}^{\nu_{2}} \delta\left(y-x_{22}\right) \partial_{\nu_{3}}^{x_{23}} \delta\left(x_{23}-x_{1 l}\right) \\
& \cdot t_{n-1}\left(\ldots,\left(\theta^{\nu_{3}} L\right)\left(x_{1 l}\right), \ldots,\left(A^{\nu_{1}} \phi^{*} \phi\right)\left(x_{21}\right),\left(\phi^{*} \phi\right)\left(x_{22}\right)\right)
\end{aligned}
$$

We see that (5.28) is separately invariant under $x_{23} \leftrightarrow y$ and that the sum $(5.29)+(5.30)$ also has this symmetry. Hence, the asserted symmetry of (5.23) holds indeed true.

So we know that

$$
\begin{aligned}
0= & \partial_{\nu_{3}}^{x_{23}} \partial_{\mu}^{y} u_{n}^{\mu}\left(L, \ldots, A^{\nu_{1}} \phi^{*} \phi, j^{\nu_{2}}, j^{\nu_{3}}\right)\left(x_{11}-y, \ldots, x_{21}-y, \ldots, x_{23}-y\right) \\
& -\left(x_{23} \leftrightarrow y\right) .
\end{aligned}
$$

Inserting the formula (5.20) for $u_{n}^{\mu \nu_{1} \nu_{2} \nu_{3}}$ into this expression and taking into account that $C_{2}=C_{3}(5.22)$, we obtain that $C_{1}=C_{2}\left(=C_{3}\right)$. Hence, also in the case $2 \mathrm{c}, u_{n}^{\mu \nu_{1} \nu_{2} \nu_{3}}$ is an admissible finite renormalization.

\footnotetext{
${ }^{11}$ We work here with a modified notation, which ignores that $t_{n+1}$ and $t_{n}$ depend on the relative coordinates only; however, it makes the computation more intelligible.
} 
Case 1: Here, $u_{n}^{\mu \nu}$ is defined by

$$
\begin{aligned}
& -\partial_{\mu}^{y} t_{n+1}(\overbrace{L, \ldots, L}^{m:=n-1}, j^{\nu}, j^{\mu})\left(x_{11}-y, \ldots, x_{1 m}-y, x_{2}-y\right) \\
& -\sum_{l=1}^{m} \partial_{\mu}^{y} \delta\left(y-x_{1 l}\right) t_{n}\left(L, \ldots,\left(\theta^{\mu} L\right), \ldots, L, j^{\nu}\right)\left(x_{11}-x_{2}, \ldots, x_{1 m}-x_{2}\right) \\
& +2 i \partial_{y}^{\nu}\left(\delta\left(y-x_{2}\right) t_{n}\left(L, \ldots, L, \phi^{*} \phi\right)\left(x_{11}-x_{2}, \ldots, x_{1 m}-x_{2}\right)\right) \\
& =:(-i)^{n} \partial_{\mu}^{y} u_{n}^{\mu \nu}\left(x_{11}-y, \ldots, x_{1 m}-y, x_{2}-y\right)
\end{aligned}
$$

where $u_{n}^{\mu \nu}$ is a Lorentz tensor of rank 2 which is a polynomial in derivatives of the $\delta$-distribution of order $\leq 2$. Taking again into account (5.17), the terms $\sim \varepsilon^{\mu \nu \alpha \beta} \partial_{\alpha}^{x_{i}} \partial_{\beta}^{x_{j}}$ are ruled out by their antisymmetry. Hence, $u_{n}^{\mu \nu}$ must be of the form

$$
\begin{aligned}
u_{n}^{\mu \nu}(\ldots)= & \left(g^{\mu \nu} \sum_{i, j} a_{i j} \partial_{i}^{\alpha} \partial_{j \alpha}+\sum_{i, j} b_{i j} \partial_{i}^{\mu} \partial_{j}^{\nu}+g^{\mu \nu} c_{0}\right) \\
= & \delta\left(x_{11}-y, \ldots, x_{1 m}-y, x_{2}-y\right) \\
& \quad \text { for some } a_{i j}, b_{i j}, c_{0} \in \mathbb{C} \text { and with } i, j \in\left\{11, \ldots, 1 m, x_{2}\right\}
\end{aligned}
$$

We have to show that $u_{n}^{\mu \nu}$ is invariant under $\left(\nu, x_{2}\right) \leftrightarrow(\mu, y)$. Obviously, for the $c_{0}$-term this holds true; hence we may omit this term in the following.

Since the 1.h.s. of (5.31) is invariant under permutations of $x_{11}, \ldots, x_{1 m}$, up to a redefinition (5.17), we may assume that $u_{n}^{\mu \nu}$ shares this permutation symmetry. We now write down all possible contributions with two derivatives to $u_{n}^{\mu \nu}$ satisfying this symmetry:

$$
\begin{array}{llll}
g^{\mu \nu} \sum_{k} \square_{k} & \sum_{k} \partial_{k}^{\mu} \partial_{k}^{\nu} & g^{\mu \nu} \sum_{k \neq l} \partial_{k}^{\alpha} \partial_{l \alpha} & \sum_{k \neq l} \partial_{k}^{\mu} \partial_{l}^{\nu}, \\
g^{\mu \nu} \partial_{2 \alpha} \sum_{k} \partial_{k}^{\alpha} & \partial_{2}^{\mu} \sum_{k} \partial_{k}^{\nu} & \partial_{2}^{\nu} \sum_{k} \partial_{k}^{\mu} & g^{\mu \nu} \square_{2} \partial_{2}^{\mu} \partial_{2}^{\nu},
\end{array}
$$

where $k, l \in\{11, \ldots, 1 m\}$. One verifies that these 9 differential operatorseach one applied to the $\delta$-distribution in (5.32) - are linearly independent if $m>1^{12}$, hence they form a basis of a vector space. We now give a different set of 9 differential operators, whose elements have a much simpler behaviour under $\left(\nu, x_{2}\right) \leftrightarrow(\mu, y)$ :

$$
\begin{aligned}
& g^{\mu \nu} \sum_{k} \square_{k} \quad \sum_{k} \partial_{k}^{\mu} \partial_{k}^{\nu} \quad \partial_{2}^{\mu} \partial_{y}^{\nu} \quad \partial_{y}^{\mu} \partial_{2}^{\nu} \quad g^{\mu \nu} \partial_{y}^{\alpha} \partial_{2 \alpha} \\
& g^{\mu \nu} \square_{2} \quad g^{\mu \nu} \square_{y} \quad \partial_{2}^{\mu} \partial_{2}^{\nu} \quad \partial_{y}^{\mu} \partial_{y}^{\nu},
\end{aligned}
$$

where again $k \in\{11, \ldots, 1 m\}$. By using

$$
\sum_{k} \partial_{k}=-\partial_{2}-\partial_{y} \quad \text { and } \quad \sum_{k \neq l} \partial_{k} \partial_{l}=\left(\sum_{k} \partial_{k}\right)^{2}-\sum_{k} \partial_{k} \partial_{k},
$$

\footnotetext{
${ }^{12}$ In the simpler case $m=1$, two of them are zero, and one may omit the first two entries of the list (1).
} 
we can express all elements of the (old) basis (5.33) as linear combination of the new terms (5.34); therefore, the latter are also a basis of the same vector space.

Under $\left(\nu, x_{2}\right) \leftrightarrow(\mu, y)$, all differential operators in the group (1) (first line of (5.34)) are individually invariant; hence, the pertinent contributions to $u_{n}^{\mu \nu}$ are admissible finite renormalizations.

To treat the remaining four terms in group (2) (second line of (5.34)), we proceed analogously to (5.23): we claim that

$\partial_{\nu}^{x_{2}} \partial_{\mu}^{y} u_{n}^{\mu \nu}\left(L, \ldots, L, j^{\nu}\right)\left(x_{11}-y, \ldots, x_{1 m}-y, x_{2}-y\right) \quad$ is invariant under $x_{2} \leftrightarrow y$

To verify this, we insert (5.31) into (5.35): obviously, the $\partial_{x_{2}} \partial_{y} t_{n+1}(\ldots, j, j)$ term and the $\partial_{x_{2}} \partial_{y}\left(\delta\left(y-x_{2}\right) t_{n}\left(\ldots, \phi^{*} \phi\right)\right)$-term fulfil the claim individually. To show this for the remaining term, we use the MWI to order $(n-1)$ :

$$
\begin{gathered}
-\sum_{l=1}^{m} \partial_{\mu}^{y} \delta\left(y-x_{1 l}\right) \partial_{\nu}^{x_{2}} t_{n}\left(L\left(x_{11}\right), \ldots,\left(\theta^{\mu} L\right)\left(x_{1 l}\right), \ldots, j^{\nu}\left(x_{2}\right)\right) \\
=\sum_{\substack{k \neq l\\
}} \partial_{\mu}^{y} \delta\left(y-x_{1 l}\right) \partial_{\nu}^{x_{2}} \delta\left(x_{2}-x_{1 k}\right) t_{n-1}\left(L\left(x_{11}\right), \ldots,\right. \\
\left.\left(\theta^{\mu} L\right)\left(x_{1 l}\right), \ldots,\left(\theta^{\nu} L\right)\left(x_{1 k}\right), \ldots, L\left(x_{1 m}\right)\right),
\end{gathered}
$$

from which we see that also this term satisfies the claim (5.35).

We conclude that the contribution from the four terms in group (2) (second line of (5.34)) satisfies

$$
0=\partial_{\nu}^{2} \partial_{\mu}^{y}\left(C_{1} g^{\mu \nu} \square_{2}+C_{2} g^{\mu \nu} \square_{y}+C_{3} \partial_{2}^{\mu} \partial_{2}^{\nu}+C_{4} \partial_{y}^{\mu} \partial_{y}^{\nu}\right)-\left(x_{2} \leftrightarrow y\right)
$$

Working out this condition, we obtain the relation

$$
C_{1}=C_{2}-C_{3}+C_{4}
$$

Thus eliminating $C_{1}$, we find that the remaining anomaly has the most general form

$$
\begin{aligned}
& d_{n}^{\nu}(\ldots) \\
& \quad=\partial_{\mu}^{y}\left(C_{2} g^{\mu \nu}\left(\square_{y}+\square_{2}\right)+C_{3}\left(\partial_{2}^{\mu} \partial_{2}^{\nu}-g^{\mu \nu} \square_{2}\right)+C_{4}\left(\partial_{y}^{\mu} \partial_{y}^{\nu}+g^{\mu \nu} \square_{2}\right)\right) \delta(\ldots) .
\end{aligned}
$$

At this point, we exploit the freedom to change $u^{\mu \nu}$ without changing $\partial_{\mu}^{y} u^{\mu \nu}$. This allows us to replace $C_{2}(\ldots)+C_{3}(\ldots)+C_{4}(\ldots)$ by

$$
\left(C_{2}+C_{4}\right) g^{\mu \nu}\left(\square_{y}+\square_{2}\right)+C_{3}\left(\partial_{2}^{\mu} \partial_{2}^{\nu}-g^{\mu \nu} \square_{2}+\partial_{y}^{\mu} \partial_{y}^{\nu}-g^{\mu \nu} \square_{y}\right),
$$

which still cancels the anomaly, and enjoys the required symmetry under $(y, \mu) \leftrightarrow\left(x_{2}, \nu\right)$. 


\section{Relation Between the Master Ward Identity and Its Simplified Version}

Our aim is to generally relate the time-ordered products $T$ and $\widehat{T}$ and to establish that the validity of the MWI for $T$ (3.7) is equivalent to the validity of the WI for $\widehat{T}(2.2)$ - to all orders and including all loop diagrams.

We assume that $B_{1}, \ldots, B_{n} \in \mathcal{P}$ are eigenvectors of $\theta(2.1)$,

$$
\theta B_{j}=b_{j} B_{j}, \quad \text { with eigenvalues } \quad b_{j} \in \mathbb{Z}, \quad \forall 1 \leq j \leq n,
$$

and that each of these field polynomials contains at most one derivated basic field, that is,

$$
\frac{\partial^{2} B_{j}}{\partial\left(\partial^{\mu} \phi\right) \partial\left(\partial^{\nu} \phi\right)}=0, \quad \frac{\partial^{2} B_{j}}{\partial\left(\partial^{\mu} \phi^{*}\right) \partial\left(\partial^{\nu} \phi\right)}=0, \quad \frac{\partial^{2} B_{j}}{\partial\left(\partial^{\mu} \phi^{*}\right) \partial\left(\partial^{\nu} \phi^{*}\right)}=0, \quad \forall 1 \leq j \leq n .
$$

Obviously these two assumptions are true for all elements of the set $\mathcal{P}_{0}$, for which we have proved the validity of the MWI in Sect. 5 .

\subsection{Complete Definition of the Finite Renormalization.}

In [11] it was investigated how the addition $i g^{\mu \nu} \delta$ to $\partial^{\mu} \partial^{\nu} \Delta^{F}$ propagates to higher orders in the inductive Epstein-Glaser construction of the sequence $\left(\widehat{T}_{n}\right)$. This was done there only for tree-like diagrams; more precisely, diagrams consisting of two components which are connected only by one internal $\phi$ line with two derivatives, and the consequences of the addition $i g^{\mu \nu} \delta$ to this line were studied. By virtue of the Main Theorem of Renormalization ([6, Eq. (3.6.25)] and Thm. A.3), we are able to give a general definition of the higher orders $\widehat{T} \equiv\left(\widehat{T}_{n}\right)$ - in particular, the inner $\phi$-line with two derivatives may be part of a loop.

In fact, we shall do more, by showing in Thm. 6.3 the equivalence of a one-parameter family of Ward identities for a family of time-ordered products $\widehat{T}_{c}$, continuously interpolating between $T=\widehat{T}_{c=0}$ and $\widehat{T}=\widehat{T}_{c=1}$. The stronger result for all $c \in \mathbb{R}$ was suggested by the analogous result [16, Sect. 3.3] found at tree-level (where all renormalizations are fixed by the time-ordered twopoint functions of the derivative fields, and the absence of anomalies can be seen explicitly) in a different but presumably equivalent setup of scalar QED, using 'string-localized' potentials.

To interpolate between the time-ordered products $T$ and $\widehat{T}$, we multiply the addition $i g^{\mu \nu} \delta(x-y)$ in the finite renormalization (2.6) by a number $c \in \mathbb{R}$, and denote the interpolating time-ordered product by $\widehat{T}_{c}$. To formulate completely the so-modified finite renormalization - in particular its consequences for the higher orders in the inductive Epstein-Glaser construction of $\widehat{T}_{c} \equiv\left(\widehat{T}_{c, n}\right)$-we work with a finite renormalization map $Z_{c} \equiv\left(Z_{c}^{(k)}\right)$, which is an element of a version of the Stückelberg-Petermann renormalization group (defined in Appendix A.2), and we will use the Main Theorem of Renormalization (given also in that Appendix in Theorem A.3).

To first order an element $Z_{c}$ of the Stückelberg-Petermann group is given by $Z_{c}^{(1)}(B(x)):=B(x)$ for all $B \in \mathcal{P}$. 
To second order, $\widehat{T}_{c, 2}$ differs from $T_{2}$ only by the finite renormalization $\partial^{\nu} \partial^{\mu} \Delta^{F} \rightarrow \partial^{\nu} \partial^{\mu} \Delta^{F}+c i g^{\mu \nu} \delta$ in the connected tree-diagram part; hence we define

$$
\begin{aligned}
Z_{c}^{(2)}\left(B_{1}\left(x_{1}\right), B_{2}\left(x_{2}\right)\right) & :=c i\left(\widehat{T}_{2}\left(B_{1}\left(x_{1}\right), B_{2}\left(x_{2}\right)\right)-T_{2}\left(B_{1}\left(x_{1}\right), B_{2}\left(x_{2}\right)\right)\right) \\
& \equiv c \zeta\left(B_{1}, B_{2}\right)\left(x_{1}\right) \delta\left(x_{1}-x_{2}\right) \\
\text { where } \quad \zeta\left(B_{1}, B_{2}\right) & :=\frac{\partial B_{1}}{\partial\left(\partial^{\mu} \phi^{*}\right)} \frac{\partial B_{2}}{\partial\left(\partial_{\mu} \phi\right)}+\frac{\partial B_{1}}{\partial\left(\partial^{\mu} \phi\right)} \frac{\partial B_{2}}{\partial\left(\partial_{\mu} \phi^{*}\right)}
\end{aligned}
$$

For later purpose, we note that

$$
\theta \zeta\left(B_{1}, B_{2}\right)=\left(b_{1}+b_{2}\right) \zeta\left(B_{1}, B_{2}\right) .
$$

For $n \geq 3$ the difference between $\widehat{T}_{c, n}$ and $T_{n}$ is only the one coming from the propagation of $Z_{c}^{(2)}$ to higher orders; hence, the higher orders of $Z_{c}$ vanish, that is,

$$
Z_{c}^{(k)}\left(B_{1}\left(x_{1}\right) \otimes \cdots \otimes B_{k}\left(x_{k}\right)\right)=0 \quad \forall k \geq 3 .
$$

We point out: $Z_{c}^{(2)}$ does not fulfil the AWI and the property 'Field Equation', because $\widehat{T}_{2, c}$ violates these relations. Comparing with the definition of the Stückelberg-Petermann group in the mentioned references, the $Z_{c}$ defined above is an element of a modified version of that group; this is explained in detail in parts A.2-A.3 of the Appendix.

We generally define $\widehat{T}_{c} \equiv\left(\widehat{T}_{c, n}\right)$ in terms of $T$ and $Z_{c}$ by using $[6$, Eq. (3.6.25)]:

$$
\begin{aligned}
i^{n} \widehat{T}_{c, n}\left(\otimes_{j=1}^{n} B_{j}\left(x_{j}\right)\right):= & i^{n} T_{n}\left(\otimes_{j=1}^{n} B_{j}\left(x_{j}\right)\right) \\
& +\sum_{\substack{P \in \operatorname{Part}_{2}(\{1, \ldots, n\}) \\
n / 2 \leq|P|<n}} i^{|P|} T_{|P|}\left(\bigotimes_{I \in P} Z_{c}^{(|I|)}\left(\otimes_{j \in I} B_{j}\left(x_{j}\right)\right)\right),
\end{aligned}
$$

where $P \in \operatorname{Part}_{2}(\{1, \ldots, n\})$ is a partition of $\{1, \ldots, n\}$ into $|P|$ disjoint subsets $I$, each of these subsets has $|I|=1$ or $|I|=2$ elements. (The latter is the reason for the subscript ' 2 ' in Part $_{2}$.) The term $|P|=n$ is explicitly written out. For $n=2$ the formula (6.8) reduces to the general definition of $Z_{c}^{(2)}$ given in (6.3).

Part (b) of the Main Theorem of Renormalization states that the sodefined $\widehat{T}_{c}$ is also a time-ordered product, that is, it satisfies the basic axioms and the renormalization conditions (v)-(viii) given in Appendix A.1; however, it may violate the AWI, the FE and any Ward identities. The proof of this statement is given in Appendix A.4.

Remark 6.1. Renormalizing the interaction $e(j A)(g)$ by the given SP renormalization map $Z_{c}$ (according to (A.11)), we indeed obtain $e(j A)(g)+c e^{2}$ $\left(A A \phi^{*} \phi\right)\left(g^{2}\right)\left(\right.$ where $\left.g^{2}(x):=(g(x))^{2}\right)$. In detail we get 


$$
\begin{aligned}
\mathcal{Z}_{c}((g, e j A)) & =e(j A)(g)+\frac{e^{2}}{2 !} \int d x_{1} d x_{2} g\left(x_{1}\right) g\left(x_{2}\right) Z_{c}^{(2)}\left((j A)\left(x_{1}\right),(j A)\left(x_{2}\right)\right) \\
& =e(j A)(g)+c e^{2}\left(A A \phi^{*} \phi\right)\left(g^{2}\right) .
\end{aligned}
$$

As explained at the end of Appendix A.2, the above definition of $\widehat{T}_{c}(6.8)$ can be written in terms of the $S$-matrix (A.4) by means of the formula (A.13): $\widehat{\mathbf{S}}_{c}:=\mathbf{S} \circ \mathcal{Z}_{c}$ by abuse of notation. For the interaction $\widetilde{L}:=e j^{\mu} A_{\mu}(2.3)$ this yields

$$
\widehat{\mathbf{S}}_{c}((g, e j A)):=\mathbf{S}\left((g, e j A),\left(g^{2}, c e^{2} A A \phi^{*} \phi\right)\right) \quad \forall g \in \mathcal{D}(\mathbb{M}),
$$

by using (6.9). For $c=1$, this is precisely the relation between $\widehat{T}$ and $T$ we want to hold-see $(2.7)$.

The physically relevant $S$-matrix for scalar QED, that is, $\mathbf{S}((g, e j A)$, $\left.\left(g^{2}, e^{2} A A \phi^{*} \phi\right)\right)$, can be expressed in terms of $\widehat{\mathbf{S}}_{c}$ by

$$
\begin{aligned}
\mathbf{S}\left((g, e j A),\left(g^{2}, e^{2} A A \phi^{*} \phi\right)\right)= & \widehat{\mathbf{S}}_{c}\left((g, e j A),\left(g^{2},(1-c) e^{2} A A \phi^{*} \phi\right)\right), \\
& \forall c \in \mathbb{R}, g \in \mathcal{D}(\mathbb{M}) .
\end{aligned}
$$

This relation is obtained by applying $\widehat{\mathbf{S}}_{c}:=\mathbf{S} \circ \mathcal{Z}_{c}$ to $\left((g, e j A),\left(g^{2},(1-\right.\right.$ c) $\left.e^{2} A A \phi^{*} \phi\right)$ ), to wit, by using the explicit formulas for $Z_{c}(6.4)-(6.7)$ we get

$$
\begin{aligned}
& \mathcal{Z}_{c}\left((g, e j A),\left(g^{2},(1-c) e^{2} A A \phi^{*} \phi\right)\right) \\
&= e(j A)(g)+(1-c) e^{2}\left(A A \phi^{*} \phi\right)\left(g^{2}\right) \\
& \quad+\frac{e^{2}}{2 !} \int d x_{1} d x_{2} g\left(x_{1}\right) g\left(x_{2}\right) Z_{c}^{(2)}\left((j A)\left(x_{1}\right),(j A)\left(x_{2}\right)\right) \\
&=e(j A)(g)+e^{2}\left(A A \phi^{*} \phi\right)\left(g^{2}\right) .
\end{aligned}
$$

Remark 6.2. (Interacting electromagnetic current in terms of $\widehat{T}$ ) Working with the time-ordered product $\widehat{T} \equiv \widehat{T}_{c=1}$, Bogoliubov's definition (4.5) of the interacting electromagnetic current reads

$$
\widehat{j_{(g, \widetilde{L}), 0}^{\mu}}(\alpha):=\left.\widehat{\mathbf{S}}((g, \widetilde{L}))_{0}^{\star-1} \star \frac{d}{i d \lambda}\right|_{\lambda=0} \widehat{\mathbf{S}}\left((g, \widetilde{L}),\left(\alpha, \lambda j^{\mu}\right)\right)_{0}, \quad g, \alpha \in \mathcal{D}(\mathbb{M}, \mathbb{R}),
$$

where $\widetilde{L}:=e j A(2.3)$. We are going to show that the definition $\widehat{\mathbf{S}}:=\mathbf{S} \circ \mathcal{Z}$ (A.13) (where $\mathcal{Z} \equiv \mathcal{Z}_{c=1}$ ) implies that

$$
\widehat{j_{(g, \widetilde{L}), 0}^{\mu}}(x)=J_{S}^{\mu}(x)_{0},
$$

where $J^{\mu}$ is given in (4.6) and $S:=e\left(j^{\mu} A_{\mu}\right)(g)+e^{2}\left(A^{2} \phi^{*} \phi\right)\left(g^{2}\right)(3.9)$. To do this we insert $\widehat{\mathbf{S}}:=\mathbf{S} \circ \mathcal{Z}$ into (6.12) and use that $\mathcal{Z}((g, e j A))=S$ (6.9). This yields

$$
\widehat{j_{(g, \widetilde{L}), 0}^{\mu}}(\alpha)=\mathbf{S}(S)_{0}^{\star-1} \star T\left(\left.e_{\otimes}^{i S} \otimes \frac{d}{d \lambda}\right|_{\lambda=0} \mathcal{Z}\left((g, \widetilde{L}),\left(\alpha, \lambda j^{\mu}\right)\right)\right)
$$


Using (A.11) and the explicit formulas for $Z$ we obtain

$$
\begin{aligned}
\left.\frac{d}{d \lambda}\right|_{\lambda=0} \mathcal{Z}\left((g, \widetilde{L}),\left(\alpha, \lambda j^{\mu}\right)\right) & =j^{\mu}(\alpha)+e \int d x_{1} d x_{2} g\left(x_{1}\right) \alpha\left(x_{2}\right) Z^{(2)}\left((j A)\left(x_{1}\right), j^{\mu}\left(x_{2}\right)\right) \\
& =j^{\mu}(\alpha)+2 e\left(A^{\mu} \phi^{*} \phi\right)(g \alpha)=J^{\mu}(\alpha) .
\end{aligned}
$$

Inserting this result into (6.14) and comparing with the definition of $J_{S}^{\mu}(\alpha)_{0}$ (4.5), we get the assertion (6.13).

The equality (6.13) can be understood in terms of Feynman diagrams: there are diagrams contributing to the second factor on the r.h.s. of (6.12), i.e. $\widehat{T}\left(\left(\otimes_{k} \widetilde{L}\left(y_{k}\right)\right) \otimes j^{\mu}(x)\right)_{0}$, in which the field vertex $x$ is connected to an interaction vertex $y_{k}$ by an internal $\phi$-line with two derivatives and this line is not part of any loop. The addition $i g^{\mu \nu} \delta$ to this line in these diagrams generates the additional term $2 e g(x)\left(A^{\mu} \phi^{*} \phi\right)_{S}(x)_{0}$ of $J_{S}^{\mu}(x)_{0}$.

From the identity (6.13) and $\partial_{\mu}^{x} J_{S}^{\mu}(x)_{0}=0$ (4.6) (that is secured by the assumed validity of the MWI for the T-products $T)$, we see that $\widehat{j_{(g, \widetilde{L}), 0}^{\mu}}$ is conserved:

$$
\partial_{\mu}^{x} \widehat{j_{(g, \widetilde{L}), 0}^{\mu}}(x)=0 .
$$

Alternatively, this result can directly be obtained, i.e. without using $J_{S}^{\mu}$; namely, from the WI for $\widehat{T}(2.2)$, by proceeding analogously to the derivation of $\partial_{\mu}^{x} J_{S}^{\mu}(x)_{0}=0$ from the MWI (4.4). Hence, with regard to the interacting electromagnetic current the WI (2.2) and the MWI (3.7) (or (4.4)) contain the same information. This result can strongly be generalized-this is the topic of the next subsection.

\subsection{The MWI for $T(3.7)$ and the WI for $\widehat{T}(2.2)$ are Equivalent}

We are now coming to the second main result of this paper.

Theorem 6.3. Given a time-ordered product $T$ and $c \in \mathbb{R}$, let a time-ordered product $\widehat{T}_{c}$ be defined in terms of $T$ and $Z_{c}$ by (6.3)-(6.5) and (6.8). Then, for all $B_{j} \in \mathcal{P}$ satisfying the assumptions (6.1) and (6.2), the validity of the $M W I$ (3.7) for $T$ is equivalent to the validity of the following c-dependent WI for $\widehat{T}_{c}$-to all orders $n \in \mathbb{N}$ :

$$
\begin{aligned}
\partial_{y}^{\mu} & \widehat{T}_{c, n+1}\left(B_{1}\left(x_{1}\right) \otimes \cdots \otimes B_{n}\left(x_{n}\right) \otimes j_{\mu}(y)\right)_{0} \\
= & \sum_{l=1}^{n} \delta\left(y-x_{l}\right) \widehat{T}_{c, n}\left(B_{1}\left(x_{1}\right) \otimes \cdots \otimes\left(\theta B_{l}\right)\left(x_{l}\right) \otimes \cdots \otimes B_{n}\left(x_{n}\right)\right)_{0} \\
& \quad+(c-1) \partial_{y}^{\mu}\left(\sum_{l=1}^{n} \delta\left(y-x_{l}\right) \widehat{T}_{c, n}\left(B_{1}\left(x_{1}\right) \otimes \cdots \otimes\left(\theta_{\mu} B_{l}\right)\left(x_{l}\right) \otimes \cdots \otimes B_{n}\left(x_{n}\right)\right)_{0}\right),
\end{aligned}
$$


For $c=1$, the assertion (6.15) agrees with the WI (2.2) and for $c=0$ with the MWI (3.7). In particular, we obtain

$\partial_{\mu}^{y} \widehat{T}_{c, 2}\left(\partial^{\nu} \phi(x) \otimes j^{\mu}(y)\right)_{0}=\delta(y-x) \partial^{\nu} \phi(x)_{0}+(c-1)\left(\partial^{\nu} \delta\right)(y-x) \phi(x)_{0}$, $\partial_{\mu}^{y} \widehat{T}_{c, 2}\left(\partial^{\nu} \phi^{*}(x) \otimes j^{\mu}(y)\right)_{0}=-\delta(y-x) \partial^{\nu} \phi^{*}(x)_{0}-(c-1)\left(\partial^{\nu} \delta\right)(y-x) \phi^{*}(x)_{0}$, $\partial_{\mu}^{y} \widehat{T}_{c, 2}\left(j^{\nu}(x) \otimes j^{\mu}(y)\right)_{0}=(1-c) 2 i\left(\phi^{*} \phi\right)(x)_{0} \partial^{\nu} \delta(y-x)$,

which contains the relations $(2.4)$ for $\widehat{T}=\widehat{T}_{c=1}$ and (3.8) for $T=\widehat{T}_{c=0}$.

Proof. $M W I$ (3.7) for $T \Longrightarrow W I$ (6.15) for $\widehat{T}_{c}$ : From (6.4)-(6.5) we obtain

$$
Z_{c}^{(2)}\left(B(x), j^{\mu}(y)\right)=i c\left(\theta^{\mu} B\right)(x) \delta(y-x)
$$

with $\theta^{\mu}$ defined in (3.4).

Using (6.8) to express $\widehat{T}_{c}$ in terms of $T$ on the 1.h.s. of (6.15), we get two types of terms: in the first type $j^{\mu}$ does not appear in the argument of any $Z_{c}^{(2)}$, in the second type it does and, hence, we may use (6.17):

$$
\begin{aligned}
& i^{n+1}[\text { l.h.s. of WI }] \\
& =\sum_{\substack{P \in \operatorname{Part}_{2}(\{1, \ldots, n\}) \\
n / 2 \leq|P| \leq n}} i^{|P|+1} \partial_{\mu}^{y} T_{|P|+1}\left(\bigotimes_{I \in P} Z_{c}^{(|I|)}\left(\otimes_{j \in I} B_{j}\left(x_{j}\right)\right), j^{\mu}(y)\right)_{0} \\
& \quad+i c \sum_{l=1}^{n}\left(\partial_{\mu} \delta\right)\left(y-x_{l}\right) \sum_{\substack{Q \in \operatorname{Part}_{2}\left(\left\{1, \ldots, \hat{l}_{, \ldots, n\})} \\
(n-1) / 2 \leq|Q| \leq n-1\right.\right.}} i^{|Q|+1} . \\
& \\
& \quad T_{|Q|+1}\left(\left(\theta^{\mu} B_{l}\right)\left(x_{l}\right) \otimes\left[\bigotimes_{I \in Q} Z_{c}^{(|I|)}\left(\otimes_{j \in I} B_{j}\left(x_{j}\right)\right)\right]\right)_{0}
\end{aligned}
$$

where $\hat{l}$ means that $l$ is omitted in the pertinent set. Now we insert the MWI (3.7) into (6.18): for the $\theta$-terms (displayed in (6.20)) we use (6.1) and (6.4)(6.6), the latter imply

$$
\begin{aligned}
\delta(y & \left.-x_{k}\right) T\left(\cdots \otimes \theta Z_{c}^{(2)}\left(B_{k}\left(x_{k}\right), B_{j}\left(x_{j}\right)\right) \otimes \cdots\right) \\
& =\delta\left(y-x_{k}, y-x_{j}\right)\left(b_{j}+b_{k}\right) T\left(\cdots \otimes c \zeta\left(B_{k}, B_{j}\right)\left(x_{k}\right) \otimes \cdots\right) \\
& =\left[\delta\left(y-x_{k}\right) b_{k}+\delta\left(y-x_{j}\right) b_{j}\right] \cdot T\left(\cdots \otimes Z_{c}^{(2)}\left(B_{k}\left(x_{k}\right), B_{j}\left(x_{j}\right)\right) \otimes \cdots\right) .
\end{aligned}
$$


For the $\theta^{\mu}$-terms (displayed in (6.21)) we take into account that $\theta^{\mu} \zeta\left(B_{1}, B_{2}\right)=$ 0 , which follows from (6.2), and we reorder the summations. So we obtain:

$$
\begin{gathered}
=i\left[\sum_{\substack{P \in \operatorname{Part}_{2}(\{1, \ldots, n\}) \\
n / 2 \leq|P| \leq n}} i^{|P|} T_{|P|}\left(\bigotimes_{I \in P} Z_{c}^{(|I|)}\left(\otimes_{j \in I} B_{j}\left(x_{j}\right)\right)\right)_{0}\right] \\
\quad \cdot\left[\sum_{l=1}^{n} \delta\left(y-x_{l}\right) b_{l}\right] \\
-i \sum_{l=1}^{n}\left(\partial_{\mu} \delta\right)\left(y-x_{l}\right) \sum_{\substack{Q \in \operatorname{Part}_{2}(\{1, \ldots, \hat{l}, \ldots, n\}) \\
(n-1) / 2 \leq|Q| \leq n-1}} i^{|Q|+1} . \\
T_{|Q|+1}\left(\left(\theta^{\mu} B_{l}\right)\left(x_{l}\right) \otimes \underset{I \in Q}{\left[\bigotimes_{c}^{(|I|)}\left(\otimes_{j \in I} B_{j}\left(x_{j}\right)\right)\right]} .\right.
\end{gathered}
$$

Finally, we reexpress $T$ in terms of $\widehat{T}_{c}$. Thanks to (6.2) and (6.4)-(6.5), it holds that

$$
Z_{c}^{(2)}\left(\left(\theta^{\mu} B_{l}\right)\left(x_{l}\right) \otimes B_{j}\left(x_{j}\right)\right)=0
$$

Hence, we obtain

$$
\begin{aligned}
& \sum_{\substack{Q \in \operatorname{Part}_{2}(\{1, \ldots, \hat{l}, \ldots, n\}) \\
(n-1) / 2 \leq|Q| \leq n-1}} i^{|Q|+1} T_{|Q|+1}\left(\left(\theta^{\mu} B_{l}\right)\left(x_{l}\right) \otimes\left[\bigotimes_{I \in Q} Z_{c}^{(|I|)}\left(\otimes_{j \in I} B_{j}\left(x_{j}\right)\right)\right]\right)_{0} \\
& =i^{n} \widehat{T}_{c, n}\left(B_{1}\left(x_{1}\right) \otimes \cdots \otimes\left(\theta^{\mu} B_{l}\right)\left(x_{l}\right) \otimes \cdots \otimes B_{n}\left(x_{n}\right)\right)_{0} .
\end{aligned}
$$

So we see that the sum of the terms (6.19) and (6.21) is equal to $i^{n+1}$. [(c-1)term on the r.h.s. of the assertion (6.15)]. And, the expression (6.20) is equal to

$$
\begin{aligned}
i^{n+1} & \widehat{T}_{c, n}\left(\otimes_{j=1}^{n} B_{j}\left(x_{j}\right)\right)_{0} \cdot\left[\sum_{l=1}^{n} \delta\left(y-x_{l}\right) b_{l}\right] \\
& =i^{n+1} \sum_{l=1}^{n} \delta\left(y-x_{l}\right) \widehat{T}_{c, n}\left(B_{1}\left(x_{1}\right) \otimes \cdots \otimes\left(\theta B_{l}\right)\left(x_{l}\right) \otimes \cdots \otimes B_{n}\left(x_{n}\right)\right)_{0},
\end{aligned}
$$

by using (6.1).

$W I$ (6.15) for $\widehat{T}_{c} \Longrightarrow M W I$ (3.7) for $T$ : First we show that for $B_{j}$ 's satisfying (6.2), the 'inverse' of $Z_{c} \in \mathcal{R}$ (see Def. A.2 for the definition of $\mathcal{R}$ ) is $Y_{c} \in \mathcal{R}$ given by $Y_{c}^{(1)}(B(x)):=B(x)$ and

$$
\begin{aligned}
& Y_{c}^{(2)}\left(B_{1}\left(x_{1}\right), B_{2}\left(x_{2}\right)\right):=-c \zeta\left(B_{1}, B_{2}\right)\left(x_{1}\right) \delta\left(x_{1}-x_{2}\right), \\
& Y_{c}^{(k)}\left(B_{1}\left(x_{1}\right), \ldots, B_{k}\left(x_{k}\right)\right):=0 \quad \forall k \geq 3 .
\end{aligned}
$$


Since $\mathcal{Z}_{c}\left(\left(g_{j}, B_{j}\right)\right)=\sum_{j} B_{j}\left(g_{j}\right)+\frac{c}{2} \sum_{j_{1}, j_{2}} \zeta\left(B_{j_{1}}, B_{j_{2}}\right)\left(g_{j_{1}} g_{j_{2}}\right)$, we have to verify the relation $\mathcal{Y}_{c}\left(\left(g_{j}, B_{j}\right),\left(g_{j_{1}} g_{j_{2}}, \frac{c}{2} \zeta\left(B_{j_{1}}, B_{j_{2}}\right)\right)\right)=\sum_{j} B_{j}\left(g_{j}\right)$, where $\mathcal{Y}_{c}$ denotes the renormalization of the interaction given by $Y_{c} \in \mathcal{R}$ according to (A.11). Taking into account that $\zeta\left(B_{j_{1}}, \zeta\left(B_{j_{2}}, B_{j_{3}}\right)\right)=0=\zeta\left(\zeta\left(B_{j_{1}}, B_{j_{2}}\right)\right.$, $\left.\zeta\left(B_{j_{3}}, B_{j_{4}}\right)\right)$ because $\frac{\partial \zeta\left(B_{j_{1}}, B_{j_{2}}\right)}{\partial\left(\partial^{\mu} \phi\right)}=0=\frac{\partial \zeta\left(B_{j_{1}}, B_{j_{2}}\right)}{\partial\left(\partial^{\mu} \phi^{*}\right)}$, we indeed obtain

$$
\begin{aligned}
& Y_{c}\left(\left(g_{j}, B_{j}\right),\left(g_{j_{1}} g_{j_{2}}, \frac{c}{2} \zeta\left(B_{j_{1}}, B_{j_{2}}\right)\right)\right) \\
& \quad=\sum_{j} B_{j}\left(g_{j}\right)+\frac{c}{2} \sum_{j_{1} \cdot j_{2}} \zeta\left(B_{j_{1}}, B_{j_{2}}\right)\left(g_{j_{1}} g_{j_{2}}\right) \\
& \quad+\frac{1}{2} \int d x_{1} d x_{2} g_{j_{1}}\left(x_{1}\right) g_{j_{2}}\left(x_{2}\right) Y_{c}^{(2)}\left(B_{j_{1}}\left(x_{1}\right), B_{j_{2}}\left(x_{2}\right)\right)=\sum_{j} B_{j}\left(g_{j}\right) .
\end{aligned}
$$

Therefore, $T_{n}$ can be expressed in terms of $\left(\widehat{T}_{c, k}\right)_{1 \leq k \leq n}$ and $Y_{c}$ by the formula (6.8): $T$ and $\widehat{T}_{c}$ are mutually exchanged and $Z_{c}$ is replaced by $Y_{c}$.

With this, the assertion (i.e. the MWI (3.7) for $T$ ) can be verified by essentially the same computation as in the above proof of the reversed statement: to compute $\partial_{\mu}^{y} T_{n+1}\left(\cdots \otimes j^{\mu}(y)\right)$ we first express $T_{n+1}$ in terms of $\widehat{T}_{c}$, then we use the WI (6.15) for $\widehat{T}_{c}$ and finally we reexpress $\widehat{T}_{c}$ in terms of $T$.

\section{Acknowledgements}

We thank the referee for reading the manuscript extremely thoroughly and pointing out a lot of improvements. M.D. profited from enlightening discussions with Klaus Fredenhagen, Romeo Brunetti and Kasia Rejzner.

Funding Open Access funding enabled and organized by Projekt DEAL.

Open Access. This article is licensed under a Creative Commons Attribution 4.0 International License, which permits use, sharing, adaptation, distribution and reproduction in any medium or format, as long as you give appropriate credit to the original author(s) and the source, provide a link to the Creative Commons licence, and indicate if changes were made. The images or other third party material in this article are included in the article's Creative Commons licence, unless indicated otherwise in a credit line to the material. If material is not included in the article's Creative Commons licence and your intended use is not permitted by statutory regulation or exceeds the permitted use, you will need to obtain permission directly from the copyright holder. To view a copy of this licence, visit http://creativecommons. org/licenses/by/4.0/.

Publisher's Note Springer Nature remains neutral with regard to jurisdictional claims in published maps and institutional affiliations. 


\section{A Stückelberg-Petermann Renormalization Group Without Action Ward Identity}

\section{A.1 Axioms for the Time-Ordered Product}

Both time-ordered products $T$ and $\widehat{T}$, used in the main text, satisfy the following definition:

Definition A.1. A time-ordered product $T$ is a sequence of maps

$$
T \equiv\left(T_{n}\right)_{n=1}^{\infty}:\left\{\begin{array}{l}
\mathcal{P}^{\otimes n} \longrightarrow \mathcal{D}^{\prime}\left(\mathbb{M}^{n}, \mathcal{F}\right) \\
B_{1} \otimes \cdots \otimes B_{n} \longmapsto T_{n}\left(B_{1}\left(x_{1}\right) \otimes \cdots \otimes B_{n}\left(x_{n}\right)\right),
\end{array}\right.
$$

fulfilling certain axioms - the basic axioms and the renormalization conditions. The former read:

(i) Linearity: $T_{n}$ is linear (that is, multilinear in $\left(B_{1}, \ldots, B_{n}\right)$ );

(ii) Initial Condition: $T_{1}(B(x))=B(x)$ for any $B \in \mathcal{P}$;

(iii) Symmetry: For all permutations $\pi$ of $(1, \ldots, n)$ it holds that

$$
T_{n}\left(B_{\pi 1}\left(x_{\pi 1}\right) \otimes \cdots \otimes B_{\pi n}\left(x_{\pi n}\right)\right)=T_{n}\left(B_{1}\left(x_{1}\right) \otimes \cdots \otimes B_{n}\left(x_{n}\right)\right) .
$$

(iv) Causality. For all $B_{1}, \ldots, B_{n} \in \mathcal{P}, T_{n}$ fulfils the causal factorization:

$T_{n}\left(B_{1}\left(x_{1}\right), \ldots, B_{n}\left(x_{n}\right)\right)=T_{k}\left(B_{1}\left(x_{1}\right), \ldots, B_{k}\left(x_{k}\right)\right) \star T_{n-k}\left(B_{k+1}\left(x_{k+1}\right), \ldots, B_{n}\left(x_{n}\right)\right)$ whenever $\left\{x_{1}, \ldots, x_{k}\right\} \cap\left(\left\{x_{k+1}, \ldots, x_{n}\right\}+\bar{V}_{-}\right)=\emptyset$ where $\bar{V}_{-}$is the closed backward lightcone.

We work with the following renormalization conditions:

(v) Field Independence:

$$
\frac{\delta T_{n}\left(B_{1}\left(x_{1}\right) \otimes \cdots \otimes B_{n}\left(x_{n}\right)\right)}{\delta \phi(z)}=\sum_{j=1}^{n} T_{n}\left(B_{1}\left(x_{1}\right) \otimes \cdots \otimes \frac{\delta B_{j}\left(x_{j}\right)}{\delta \phi(z)} \otimes \cdots \otimes B_{n}\left(x_{n}\right)\right)
$$

and similarly for $\frac{\delta}{\delta \phi^{*}(z)}$ and $\frac{\delta}{\delta A^{\mu}(z)}$. This axiom is equivalent to the requirement that $T_{n}$ satisfies the causal Wick expansion, which is a unique prescription for the expansion of the time-ordered product in terms of Wick products. To wit, for monomials $B_{1}, \ldots, B_{n} \in \mathcal{P}$ it holds that

$$
T_{n}\left(B_{1}\left(x_{1}\right), \ldots, B_{n}\left(x_{n}\right)\right)=\sum_{\underline{B}_{l} \subset B_{l}} \omega_{0}\left(T_{n}\left(\underline{B}_{1}\left(x_{1}\right), \ldots, \underline{B}_{n}\left(x_{n}\right)\right)\right) \bar{B}_{1}\left(x_{1}\right) \cdots \bar{B}_{n}\left(x_{n}\right),
$$

where the submonomials $\underline{B}$ of a given monomial $B \in \mathcal{P}$ of order $n$ and their complementary submonomials $\bar{B}$ are defined by

$$
\begin{aligned}
& \underline{B}:=\frac{\partial^{k} B}{\partial \varphi_{a_{1}} \cdots \partial \varphi_{a_{k}}} \neq 0, \\
& \bar{B}:=C_{a_{1} \ldots a_{k}} \varphi_{a_{1}} \cdots \varphi_{a_{k}} \quad\left(\text { no sum over } a_{1}, \ldots, a_{k}\right),
\end{aligned}
$$

where each $C_{a_{1} \ldots a_{k}}$ is a certain combinatorial factor. The range of the sum $\sum_{\underline{B} \subset B}$ are all allowable $k \leq n$ and indices $a_{1}, \ldots, a_{k}$ of the fields $\varphi_{a}=A^{\underline{\mu}}, \phi, \phi^{*}, \partial^{\mu} \phi$ and $\partial^{\nu} \phi^{*}$ generating $\mathcal{P}$. (For $k=0$ we have $\underline{B}=B$ and $\bar{B}=1$.) 
(vi) *-Structure: To formulate this axiom, we introduce the $S$-matrix to the interaction

$$
\sum_{j=1}^{J} B_{j}\left(g_{j}\right) \equiv \sum_{j=1}^{J} \int d x B_{j}(x) g_{j}(x), \quad B_{j} \in \mathcal{P}, g_{j} \in \mathcal{D}(\mathbb{M})
$$

it is the generating functional of the time-ordered products, understood as a formal series in the $g_{j}$ 's:

$$
\begin{aligned}
& \mathbf{S}\left(\left(g_{j}, B_{j}\right)_{j=1}^{J}\right) \\
& :=1+\sum_{n=1}^{\infty} \frac{i^{n}}{n !} \int d x_{1} \cdots d x_{n} \\
& \quad \sum_{j_{1}, \ldots, j_{n}=1}^{J} g_{j_{1}}\left(x_{1}\right) \cdots g_{j_{n}}\left(x_{n}\right) T_{n}\left(B_{j_{1}}\left(x_{1}\right), \ldots, B_{j_{n}}\left(x_{n}\right)\right) .
\end{aligned}
$$

The axiom $*$-Structure reads

$$
\mathbf{S}\left(\left(g_{j}, B_{j}\right)_{j=1}^{J}\right)^{*}=\mathbf{S}\left(\left(\overline{g_{j}}, B_{j}^{*}\right)_{j=1}^{J}\right)^{\star-1}
$$

where $G^{\star-1}$ is the inverse w.r.t. the star product of $G \in \mathcal{F}$. For a real interaction (i.e. $\overline{g_{j}}=g_{j}, B_{j}^{*}=B_{j}$ for all $j$ ) this axiom asserts the unitarity of the $S$-matrix w.r.t. the star product (and as a formal series).

(vii) Poincaré Covariance:

$$
\begin{aligned}
& \beta_{\Lambda, a} T_{n}\left(B_{1}\left(x_{1}\right) \otimes \cdots \otimes B_{n}\left(x_{n}\right)\right)=T_{n}\left(\beta_{\Lambda, a} B_{1}\left(x_{1}\right) \otimes \cdots \otimes \beta_{\Lambda, a} B_{n}\left(x_{n}\right)\right) \\
& \quad \forall(\Lambda, a) \in \mathcal{P}_{+}^{\uparrow},
\end{aligned}
$$

where $(\Lambda, a) \mapsto \beta_{\Lambda, a}$ is the natural representation of $\mathcal{P}_{+}^{\uparrow}$ on $\mathcal{F}$ (see $[6$, Chap. 3.1.4]). An immediate consequence of translation covariance is that the $\mathbb{C}$-valued distributions

$$
t_{n}\left(B_{1}, \ldots, B_{n}\right)\left(x_{1}-x_{n}, \ldots\right):=\omega_{0}\left(T_{n}\left(B_{1}\left(x_{1}\right) \otimes \cdots \otimes B_{n}\left(x_{n}\right)\right)\right) \in \mathcal{D}^{\prime}\left(\mathbb{M}^{n-1}, \mathbb{C}\right)
$$

depend only on the relative coordinates.

(viii) Scaling Degree: With sd $t$ denoting the scaling degree of the distribution $t$ w.r.t. the origin (see e.g. [6, Def. 3.2.5]) the $\operatorname{VEVs}($ A.5) are required to fulfil

$$
\operatorname{sd} t_{n}\left(B_{1}, \ldots, B_{n}\right)\left(x_{1}-x_{n}, \ldots\right) \leq \sum_{j=1}^{n} \operatorname{dim} B_{j}
$$

for all $B_{1}, \ldots, B_{n} \in \mathcal{P}_{\text {hom }}$, where $\operatorname{dim} B$ is the mass dimension of $B$ and $\mathcal{P}_{\text {hom }}$ is the subset of $\mathcal{P}$ of all field polynomials being homogeneous in the mass dimension (see [6, Chap. 3.1.5]).

The time-ordered product $T$, underlying Sects. 3, and 5, fulfils additionally the following two renormalization conditions. 


\section{AWI Action Ward Identity:}

$$
\begin{aligned}
& \partial_{x_{j}} T_{n}\left(B_{1}\left(x_{1}\right) \otimes \cdots \otimes B_{j}\left(x_{j}\right) \otimes \cdots\right) \\
& \quad=T_{n}\left(B_{1}\left(x_{1}\right) \otimes \cdots \otimes \partial_{x_{j}} B_{j}\left(x_{j}\right) \otimes \cdots\right) \quad \forall 1 \leq j \leq n,
\end{aligned}
$$

which implies that $T_{n}$ can be interpreted as a map $T_{n}: \mathcal{F}_{\text {loc }}^{\otimes n} \rightarrow \mathcal{F}$ (or $T_{n}: \mathcal{F}_{\text {loc }}^{\otimes_{\mathrm{s}} n} \rightarrow \mathcal{F}$ due to the axiom Symmetry, with $\otimes_{\mathrm{s}}$ denoting the symmetrized tensor product); for details see [6, Chap. 3.1.1]. For $F_{k}=\sum_{j_{k}} \int d x g_{j_{k}}(x) B_{j_{k}}(x) \in \mathcal{F}_{\text {loc }}, g_{j_{k}} \in \mathcal{D}(\mathbb{M}), B_{j_{k}} \in \mathcal{P}$; the two kinds of maps $T_{n}$ are related by

$$
\begin{aligned}
& T_{n}\left(F_{1} \otimes \cdots \otimes F_{n}\right) \\
& \quad=\sum_{j_{1}, \ldots, j_{n}} \int d x_{1} \cdots d x_{n} g_{j_{1}}\left(x_{1}\right) \cdots g_{j_{n}}\left(x_{n}\right) T_{n}\left(B_{j_{1}}\left(x_{1}\right) \otimes \cdots \otimes B_{j_{n}}\left(x_{n}\right)\right) .
\end{aligned}
$$

FE Field Equation:

$$
\begin{aligned}
& T_{n+1}\left(\partial^{a} \phi(x) \otimes B_{1}\left(x_{1}\right) \otimes \cdots \otimes B_{n}\left(x_{n}\right)\right) \\
&= \partial^{a} \phi(x) T_{n}\left(B_{1}\left(x_{1}\right) \otimes \cdots \otimes B_{n}\left(x_{n}\right)\right) \\
& \quad+\int d y \partial^{a} \Delta^{F}(x-y) \frac{\delta}{\delta \phi^{*}(y)} T_{n}\left(B_{1}\left(x_{1}\right) \otimes \cdots \otimes B_{n}\left(x_{n}\right)\right)
\end{aligned}
$$

and analogously for $\phi$ replaced by $\phi^{*}$ or $A^{\mu}$.

In the inductive step of the Epstein-Glaser construction of the sequence $\left(T_{n}\right)$, the basic axioms determine $T_{n}\left(B_{1}\left(x_{1}\right) \otimes \cdots \otimes B_{n}\left(x_{n}\right)\right)$ uniquely on $\mathcal{D}\left(\mathbb{M}^{n} \backslash\right.$ $\left.\Delta_{n}\right)$ in terms of the $T_{k}$ of lower orders $1 \leq k<n\left(\right.$ for all $\left.B_{1}, \ldots, B_{n} \in \mathcal{P}\right)$, where

$$
\Delta_{n}:=\left\{\left(x_{1}, \ldots, x_{n}\right) \in \mathbb{M}^{n} \mid x_{1}=x_{2}=\ldots=x_{n}\right\}
$$

is the thin diagonal. The extension of $T_{n}\left(B_{1}\left(x_{1}\right) \otimes \cdots \otimes B_{n}\left(x_{n}\right)\right)$ to $\mathcal{D}\left(\mathbb{M}^{n}\right)$ is in general nonunique, it is named 'renormalization', because it corresponds to what is 'renormalization' in conventional approaches. The only purpose of the further axioms (v)-(viii) (and possibly AWI and FE), is to restrict this extension; therefore, they are called 'renormalization conditions'. They also give some indications how to do the extension: thanks to the causal Wick expansion and translation covariance, the extension is done in terms of the numerical distributions $t_{n}$ (A.5); hence the problem of renormalization is reduced to the extension of $\mathbb{C}$-valued distributions to one point, to wit, the extension from $\mathcal{D}^{\prime}\left(\mathbb{M}^{n-1} \backslash\{0\}, \mathbb{C}\right)$ to $\mathcal{D}^{\prime}\left(\mathbb{M}^{n-1}, \mathbb{C}\right)$. Two extensions $t_{n, 1}$ and $t_{n, 2}$ of $t_{n} \in \mathcal{D}^{\prime}\left(\mathbb{M}^{n-1} \backslash\{0\}, \mathbb{C}\right)$ differ by a finite linear combination of derivatives of the $\delta$-distribution, the order of the derivatives being bounded by the renormalization condition Scaling Degree:

$$
t_{n, 2}(x)-t_{n, 1}(x)=\sum_{|a|=0}^{\omega} C_{a} \partial_{x}^{a} \delta(x), \quad \text { where } \quad \omega \equiv \omega\left(t_{n}\right):=\operatorname{sd}\left(t_{n}\right)-4(n-1)
$$

is called the 'singular order' of $t_{n}$. The coefficients $C_{a} \in \mathbb{C}$ are restricted by the further renormalization conditions. 


\section{A.2 Stückelberg-Petermann Renormalization Group $\mathcal{R}$ and Main Theorem of Renormalization}

In this paper, we work with that version of the Stückelberg-Petermann renormalization group (SP-RG) that describes finite renormalizations of time-ordered products satisfying the renormalization conditions (v)-(viii) given in the preceding Sect., however, they may violate the AWI and the FE. In the absence of the AWI, the arguments of the elements of the SP-RG cannot be written as local functionals, as it is done in $[3,10]$ and [6, Chap. 3.6].

Definition A.2. The Stückelberg-Petermann renormalization group is the set $\mathcal{R}$ of all sequences of maps ${ }^{13}$

$$
Z \equiv\left(Z^{(n)}\right)_{n=1}^{\infty}:\left\{\begin{array}{l}
\mathcal{P}^{\otimes n} \longrightarrow \mathcal{D}^{\prime}\left(\mathbb{M}^{n}, \mathcal{F}_{\text {loc }}\right) \\
B_{1} \otimes \cdots \otimes B_{n} \longmapsto Z^{(n)}\left(B_{1}\left(x_{1}\right) \otimes \cdots \otimes B_{n}\left(x_{n}\right)\right)
\end{array}\right.
$$

being linear (that is, multilinear in $\left.\left(B_{1}, \ldots, B_{n}\right)\right)$ and symmetric in the sense that

$$
Z^{(n)}\left(B_{\pi 1}\left(x_{\pi 1}\right) \otimes \cdots \otimes B_{\pi n}\left(x_{\pi n}\right)\right)=Z^{(n)}\left(B_{1}\left(x_{1}\right) \otimes \cdots \otimes B_{n}\left(x_{n}\right)\right)
$$

for all permutations $\pi$ of $(1, \ldots, n)$. In addition, the maps $Z^{(n)}$ are required to satisfy the following properties for all $B, B_{1}, \ldots, B_{n} \in \mathcal{P}$ and for all $n \geq 1$ :

(1) Lowest order: $Z^{(1)}(B(x))=B(x)$.

(2) Locality: the support (in the sense of distributions) of every $Z^{(n)}\left(B_{1}\left(x_{1}\right) \otimes\right.$ ...) lies on the thin diagonal (A.6), that is,

$$
\operatorname{supp} Z^{(n)}\left(B_{1}\left(x_{1}\right) \otimes \cdots \otimes B_{n}\left(x_{n}\right)\right) \subseteq \Delta_{n} .
$$

(3) Field Independence:

$\frac{\delta Z^{(n)}\left(B_{1}\left(x_{1}\right) \otimes \cdots \otimes B_{n}\left(x_{n}\right)\right)}{\delta \phi(z)}=\sum_{j=1}^{n} Z^{(n)}\left(B_{1}\left(x_{1}\right) \otimes \cdots \otimes \frac{\delta B_{j}\left(x_{j}\right)}{\delta \phi(z)} \otimes \cdots \otimes B_{n}\left(x_{n}\right)\right)$

and similarly for $\frac{\delta}{\delta \phi^{*}(z)}$ and $\frac{\delta}{\delta A^{\mu}(z)}$. This property is equivalent to the validity of the (causal) Wick expansion for $Z^{(n)}$.

(4) Poincaré Covariance:

$$
\begin{aligned}
& \beta_{\Lambda, a} Z^{(n)}\left(B_{1}\left(x_{1}\right) \otimes \cdots \otimes B_{n}\left(x_{n}\right)\right) \\
& \quad=Z^{(n)}\left(\beta_{\Lambda, a} B_{1}\left(x_{1}\right) \otimes \cdots \otimes \beta_{\Lambda, a} B_{n}\left(x_{n}\right)\right) \quad \forall(\Lambda, a) \in \mathcal{P}_{+}^{\uparrow} .
\end{aligned}
$$

(5) *-Structure:

$$
Z^{(n)}\left(B_{1}\left(x_{1}\right) \otimes \cdots \otimes B_{n}\left(x_{n}\right)\right)^{*}=Z^{(n)}\left(B_{1}^{*}\left(x_{1}\right) \otimes \cdots \otimes B_{n}^{*}\left(x_{n}\right)\right) .
$$

(6) Scaling Degree: introducing

$$
\begin{aligned}
z^{(n)}\left(B_{1}, \ldots, B_{n}\right)\left(x_{1}-x_{n}, \ldots\right) & :=\omega_{0}\left(Z^{(n)}\left(B_{1}\left(x_{1}\right) \otimes \cdots \otimes B_{n}\left(x_{n}\right)\right)\right) \\
& \in \mathcal{D}^{\prime}\left(\mathbb{M}^{n-1}, \mathbb{C}\right)
\end{aligned}
$$

\footnotetext{
${ }^{13}$ Mind the difference: $Z^{(n)}$ takes values in the $\mathcal{F}_{\text {loc }}$-valued distributions -in contrast to $T_{n}$.
} 
in analogy to $t_{n}(\mathrm{~A} .5)$, the condition is that

$$
\operatorname{sd} z^{(n)}\left(B_{1}, \ldots, B_{n}\right)\left(x_{1}-x_{n}, \ldots\right) \leq \sum_{j=1}^{n} \operatorname{dim} B_{j}
$$

for all $B_{1}, \ldots, B_{n} \in \mathcal{P}_{\text {hom }}$.

From the property 'Locality' it follows that $\operatorname{supp} z^{(n)}\left(B_{1}, \ldots, B_{n}\right)\left(x_{1}-\right.$ $\left.x_{n}, \ldots\right) \subseteq\{(0, \ldots, 0)\}$ and taking also into account the property Scaling Degree we conclude that

$$
\begin{aligned}
& z^{(n)}\left(B_{1}, \ldots, B_{n}\right)\left(x_{1}-x_{n}, \ldots\right) \\
& =\sum_{|a|=0}^{\omega\left(B_{1}, \ldots, B_{n}\right)} C_{a}\left(B_{1}, \ldots, B_{n}\right) \partial^{a} \delta\left(x_{1}-x_{n}, \ldots, x_{n-1}-x_{n}\right), \\
& \quad \text { with } \omega\left(B_{1}, \ldots, B_{n}\right):=\sum_{j=1}^{n} \operatorname{dim} B_{j}-4(n-1)
\end{aligned}
$$

and some coefficients $C_{a}\left(B_{1}, \ldots, B_{n}\right) \in \mathbb{C}$ depending on $B_{1}, \ldots, B_{n}$.

Denoting the coupling constant by $\kappa$ (e.g., $\kappa=e$ for scalar QED), let $\sum_{j=1}^{J} B_{j}\left(g_{j}\right) \in \mathcal{F}_{\text {loc }} \llbracket \kappa, \hbar \rrbracket^{14}$ (with $B_{j} \in \mathcal{P} \llbracket \kappa, \hbar \rrbracket$ and $g_{j} \in \mathcal{D}(\mathbb{M})$ ) be the interaction, a typical example is $S=e\left(j^{\mu} A_{\mu}\right)(g)+e^{2}\left(A^{\mu} A_{\mu} \phi^{*} \phi\right)\left(g^{2}\right)(3.9)$. The renormalization of this interaction given by the sequence of maps $Z \equiv\left(Z^{(n)}\right) \in \mathcal{R}$ is defined by their generating functional

$$
\begin{aligned}
\mathcal{Z}\left(\left(g_{j}, B_{j}\right)_{j=1}^{J}\right):= & \sum_{j=1}^{J} B_{j}\left(g_{j}\right) \\
& +\sum_{n=2}^{\infty} \frac{1}{n !} \int d x_{1} \cdots d x_{n} \\
& \sum_{j_{1}, \ldots, j_{n}=1}^{J} g_{j_{1}}\left(x_{1}\right) \cdots g_{j_{n}}\left(x_{n}\right) Z^{(n)}\left(B_{j_{1}}\left(x_{1}\right), \ldots, B_{j_{n}}\left(x_{n}\right)\right) \\
=: & \sum_{k=1}^{K} P_{k}\left(f_{k}\right) \in \mathcal{F}_{\text {loc }} \llbracket \kappa, \hbar \rrbracket,
\end{aligned}
$$

by integrating out the $\delta$-distributions appearing in (A.10), where $P_{k} \in \mathcal{P} \llbracket \kappa, \hbar \rrbracket$ and $f_{k} \in \mathcal{D}(\mathbb{M})$ are uniquely determined.

The Main Theorem of Renormalization is due to Popineau and Stora [14]; the more elaborated version given here is essentially taken from [10], see also [6, Chap. 3.6.1-2] and [3]. In the formalism at hand, it can be formulated as follows:

Theorem A.3. (Main Theorem of Renormalization)

\footnotetext{
${ }^{14} \mathcal{F}_{\text {loc }} \llbracket \kappa, \hbar \rrbracket$ and $\mathcal{P} \llbracket \kappa, \hbar \rrbracket$ are the vector spaces of formal power series in the coupling constant $\kappa$ and in $\hbar$, with coefficients in $\mathcal{F}_{\text {loc }}$ and in $\mathcal{P}$, respectively.
} 
(a) Given two time-ordered products $T=\left(T_{n}\right)$ and $\widehat{T}=\left(\widehat{T}_{n}\right)$ (with generating functionals $\mathbf{S}$ and $\widehat{\mathbf{S}}$, resp.) both fulfilling the axioms (i)-(viii), there exists a unique renormalization map $Z \in \mathcal{R}$ fulfilling

$$
\widehat{\mathbf{S}}\left(\left(g_{j}, B_{j}\right)_{j=1}^{J}\right)=\mathbf{S}\left(\left(f_{k}, P_{k}\right)_{k=1}^{K}\right), \quad \forall B_{j} \in \mathcal{P} \llbracket \kappa, \hbar \rrbracket, g_{j} \in \mathcal{D}(\mathbb{M}), \quad J \in \mathbb{N},
$$

where $\left(f_{k}, P_{k}\right)_{k=1}^{K}$ is defined in terms of $\left(g_{j}, B_{j}\right)_{j=1}^{J}$ and $Z$ according to (A.11).

(b) Conversely, given a time-ordered product $T$ fulfilling the axioms (i)-(viii) and an arbitrary $Z \in \mathcal{R}$, the sequence of maps $\widehat{T} \equiv\left(\widehat{T}_{n}\right)_{n=1}^{\infty}$ defined by (A.12) (written concisely in (A.13)) satisfies also the axioms (i)-(viii) for a time-ordered product.

Interpreting, by abuse of notation, the renormalization of the interaction $\mathcal{Z}$ (A.11) as the map $\mathcal{Z}:\left(g_{j}, B_{j}\right)_{j=1}^{J} \rightarrow\left(f_{k}, P_{k}\right)_{k=1}^{K}$, the crucial relation (A.12) can be written in a more catchy form as

$$
\widehat{\mathbf{S}}=\mathbf{S} \circ \mathcal{Z}
$$

If one selects from the relation (A.12) the terms of order $n$ in the $B_{j}$ 's for a $Z \in \mathcal{R}$ satisfying $Z^{(k)}=0 \forall k \geq 3$ (as it holds for $Z_{c}(6.7)$ ), then one obtains precisely the equation (6.8).

In this paper, we only prove part (b) of this Theorem and only for the particular family of elements $Z_{c}$ of the SP-RG, given in $(6.3)-(6.7)$; this is done in section A.4.

\section{A.3 Verification That the Concretely Given $Z_{c}$ Lies in $\mathcal{R}$}

$Z_{c}^{(1)}$ is uniquely determined by the defining property (1) of the SP-RG $\mathcal{R}$. In this section, we verify that $Z_{c}^{(2)}$, concretely given in (6.3)-(6.5), satisfies the defining properties for $Z_{c}^{(n)}$ given above; this implies then that $Z_{c}:=$ $\left(Z_{c}^{(1)}, Z_{c}^{(2)}, 0,0, \ldots\right)$ lies indeed in $\mathcal{R}$.

Obviously, for any $h \in \mathcal{D}\left(\mathbb{M}^{2}\right)$ it holds that

$$
\begin{aligned}
& \int d x_{1} d x_{2} h\left(x_{1}, x_{2}\right) Z_{c}^{(2)}\left(B_{1}\left(x_{1}\right) \otimes B_{2}\left(x_{2}\right)\right) \\
& =c \int d x h(x, x) \zeta\left(B_{1}, B_{2}\right)(x) \text { lies in } \mathcal{F}_{\text {loc }}
\end{aligned}
$$

Linearity, Symmetry (A.9) and Locality (defining property (2)) of $Z_{c}^{(2)}$ are obvious. 
To provethe property Field Independence of $Z_{c}^{(2)}$ (defining property (3)), we use the assumption (6.2), which, e.g., implies $\frac{\delta}{\delta \phi(z)} \frac{\partial B_{j}}{\partial\left(\partial^{\mu} \phi\right)}\left(x_{1}\right)=\frac{\partial^{2} B_{j}}{\partial\left(\partial^{\mu} \phi\right) \partial \phi}\left(x_{1}\right)$ $\delta\left(x_{1}-z\right)$ :

$$
\begin{aligned}
& \frac{\delta Z_{c}^{(2)}\left(B_{1}\left(x_{1}\right) \otimes B_{2}\left(x_{2}\right)\right)}{\delta \phi(z)} \\
& \quad=c \delta\left(x_{1}-x_{2}, x_{1}-z\right)\left(\frac{\partial^{2} B_{1}}{\partial\left(\partial^{\mu} \phi^{*}\right) \partial \phi} \frac{\partial B_{2}}{\partial\left(\partial_{\mu} \phi\right)}+\frac{\partial^{2} B_{1}}{\partial\left(\partial^{\mu} \phi\right) \partial \phi} \frac{\partial B_{2}}{\partial\left(\partial_{\mu} \phi^{*}\right)}+\left(B_{1} \leftrightarrow B_{2}\right)\right)\left(x_{1}\right) \\
& \quad=\delta\left(x_{1}-z\right) Z_{c}^{(2)}\left(\frac{\partial B_{1}}{\partial \phi}\left(x_{1}\right) \otimes B_{2}\left(x_{2}\right)\right)+\delta\left(x_{2}-z\right) Z_{c}^{(2)}\left(B_{1}\left(x_{1}\right) \otimes \frac{\partial B_{2}}{\partial \phi}\left(x_{2}\right)\right) \\
& \quad=Z_{c}^{(2)}\left(\frac{\delta B_{1}\left(x_{1}\right)}{\delta \phi(z)} \otimes B_{2}\left(x_{2}\right)\right)+Z_{c}^{(2)}\left(B_{1}\left(x_{1}\right) \otimes \frac{\delta B_{2}\left(x_{2}\right)}{\delta \phi(z)}\right)
\end{aligned}
$$

and similarly for $\frac{\delta}{\delta \phi^{*}(z)}$ and $\frac{\delta}{\delta A^{\mu}(z)}$. In the last step we have used again (6.2) to conclude that e.g. the term $\frac{\partial B_{1}}{\partial\left(\partial^{\nu} \phi\right)}\left(x_{1}\right) \partial^{\nu} \delta\left(x_{1}-z\right)$ of $\frac{\delta B_{1}\left(x_{1}\right)}{\delta \phi(z)}$ does not contribute to $Z_{c}^{(2)}\left(\frac{\delta B_{1}\left(x_{1}\right)}{\delta \phi(z)} \otimes B_{2}\left(x_{2}\right)\right)$.

Poincaré Covariance (defining property (4)): Translation covariance of $Z_{c}^{(2)}$ is obvious and Lorentz covariance follows from the fact that $\zeta\left(B_{1}, B_{2}\right)\left(x_{1}\right)$ is a Lorentz tensor of the same type as $B_{1}\left(x_{1}\right) B_{2}\left(x_{2}\right)$.

The property $*$-Structure of $Z_{c}^{(2)}$ (defining property (5)) follows from $\zeta\left(B_{1}, B_{2}\right)^{*}=\zeta\left(B_{1}^{*}, B_{2}^{*}\right)$, which relies on $\left(\frac{\partial B}{\partial\left(\partial^{\mu} \phi\right)}\right)^{*}=\frac{\partial B^{*}}{\partial\left(\partial^{\mu} \phi^{*}\right)}$.

To verify the property Scaling Degree (defining property (6)) note first that $z_{c}^{(2)}\left(B_{1}, B_{2}\right)$ is non-vanishing only for $\left(B_{1}, B_{2}\right)=\left(\partial^{\mu} \phi, \partial^{\nu} \phi^{*}\right)$ or $\left(B_{1}, B_{2}\right)=$ $\left(\partial^{\nu} \phi^{*}, \partial^{\mu} \phi\right)$. In both cases, it holds that $\zeta\left(B_{1}, B_{2}\right)=g^{\mu \nu}$, so we obtain

$$
\operatorname{sd} z_{c}^{(2)}\left(B_{1}, B_{2}\right)(y)=\operatorname{sd}\left(g^{\mu \nu} \delta(y)\right)=4=\operatorname{dim} \partial^{\mu} \phi+\operatorname{dim} \partial^{\nu} \phi^{*} .
$$

\section{A.4 Proof That $\widehat{T}_{c}$ Constructed from $T$ and $Z_{c}$ by (6.8) is a Time-Ordered Product}

In this section, we prove that $\widehat{T}_{c}$, defined in (6.8) in terms of $T$ and the concretly given $Z_{c}$, satisfies the basic axioms (i)-(iv) and the renormalization conditions (v)-(viii) given in Appendix A.1. This statement is part (b) of the Main Theorem for the particular $Z_{c}$ given in (6.3)-(6.7). Since, in contrast to [6, Chapt. 3.6.1-2] and [3,10], we are forced to work in a formalism not fulfilling the AWI, we cannot refer to the general proof of the Main Theorem given in these references.

Basic axioms. The Initial Condition (ii), $\widehat{T}_{c, 1}(B(x))=B(x)$, is obvious. Linearity (i) in $B_{1} \otimes \cdots \otimes B_{n}$ and Symmetry (iii) follow from the corresponding properties of $T$ and $Z_{c}$, as we see by looking at (6.8).

To verify Causality (iv) let $\left\{x_{1}, \ldots, x_{k}\right\} \cap\left(\left\{x_{k+1}, \ldots, x_{n}\right\}+\bar{V}_{-}\right)=\emptyset$. By Locality of $Z_{c}^{(2)}$ (defining property (2)) it holds that

$$
Z_{c}^{(2)}\left(B_{j}\left(x_{j}\right) \otimes B_{l}\left(x_{l}\right)\right)=0 \quad \text { if } 1 \leq j \leq k \text { and } k+1 \leq l \leq n .
$$


Using this and in a second step Causality (iv) of $T$ we indeed obtain causal factorization of $\widehat{T}_{c, n}$, in detail:

$$
\begin{aligned}
i^{n} \widehat{T}_{c, n}\left(\otimes_{j=1}^{n} B_{j}\left(x_{j}\right)\right) & \sum_{\substack{P \in \operatorname{Part}_{2}(\{1, \ldots, k\}) \\
k / 2 \leq|P| \leq k}} \sum_{\substack{Q \in \operatorname{Part}_{2}(\{k+1, \ldots, n\}) \\
(n-k) / 2 \leq|Q| \leq n-k}} i^{|P|+|Q|} \\
& \cdot T_{|P|+|Q|}\left(\bigotimes_{I \in P} Z_{c}^{(|I|)}\left(\otimes_{j \in I} B_{j}\left(x_{j}\right)\right) \otimes \bigotimes_{R \in Q} Z_{c}^{(|R|)}\left(\otimes_{r \in R} B_{r}\left(x_{r}\right)\right)\right) \\
= & \sum_{\substack{P \in \operatorname{Part}_{2}(\{1, \ldots, k\}) \\
k / 2 \leq|P| \leq k}} i^{|P|} T_{|P|}\left(\bigotimes_{I \in P} Z_{c}^{(|I|)}\left(\otimes_{j \in I} B_{j}\left(x_{j}\right)\right)\right) \\
& \star \sum_{\substack{Q \in \operatorname{Part}_{2}(\{k+1, \ldots, n\}) \\
(n-k) / 2 \leq|Q| \leq n-k}} i^{|Q|} T_{|Q|}\left(\bigotimes_{R \in Q} Z_{c}^{(|R|)}\left(\otimes_{r \in R} B_{r}\left(x_{r}\right)\right)\right) \\
= & i^{n} \widehat{T}_{c, k}\left(\otimes_{j=1}^{k} B_{j}\left(x_{j}\right)\right) \star \widehat{T}_{c, n-k}\left(\otimes_{r=k+1}^{n} B_{r}\left(x_{r}\right)\right) .
\end{aligned}
$$

Renormalization conditions. The validity of Field Independence (v) and Poincaré Covariance (vii) for $\widehat{T}_{c}$ follows straightforwardly from the corresponding properties of $T$ and $Z_{c}$.

To verify the axiom (vi) *-Structure for $\widehat{T}_{c}$, we first conclude from the property *-Structure of $Z_{c}$ (defining property (5)) and (A.11) that, if $\mathcal{Z}_{c}\left(\left(g_{j}, B_{j}\right)_{j}\right)=\left(f_{k}, P_{k}\right)_{k}$ (by abuse of notation), then $\mathcal{Z}_{c}\left(\left(\overline{g_{j}}, B_{j}^{*}\right)_{j}\right)=\left(\overline{f_{k}}, P_{k}^{*}\right)_{k}$. With this and by using (A.13) (or (A.12), resp.) and the axiom *-Structure for $T$, we obtain

$$
\begin{aligned}
& \widehat{\mathbf{S}}_{c}\left(\left(\overline{g_{j}}, B_{j}^{*}\right)_{j}\right)^{\star-1}=\mathbf{S}\left(\mathcal{Z}_{c}\left(\left(\overline{g_{j}}, B_{j}^{*}\right)_{j}\right)\right)^{\star-1} \\
& \quad=\mathbf{S}\left(\left(\overline{f_{k}}, P_{k}^{*}\right)_{k}\right)^{\star-1}=\mathbf{S}\left(\left(f_{k}, P_{k}\right)_{k}\right)=\widehat{\mathbf{S}}_{c}\left(\left(g_{j}, B_{j}\right)_{j}\right) .
\end{aligned}
$$

To prove that $\widehat{T}_{c}$ satisfies the axiom (viii) Scaling Degree, first note that, up to permutations of $\left(B_{1}\left(x_{1}\right), \ldots, B_{n}\left(x_{n}\right)\right)$ and the prefactor $i^{|P|}$, every summand of

$$
\begin{aligned}
& \widehat{t}_{c, n}\left(B_{1}, \ldots, B_{n}\right)\left(x_{1}-x_{n}, \ldots\right) \\
& =\sum_{\substack{P \in \operatorname{Part}_{2}(\{1, \ldots, n\}) \\
n / 2 \leq|P| \leq n}} i^{|P|} \omega_{0}\left(T_{|P|}\left(\bigotimes_{I \in P} Z_{c}^{(|I|)}\left(\otimes_{j \in I} B_{j}\left(x_{j}\right)\right)\right)\right)
\end{aligned}
$$


is equal to

$$
\begin{aligned}
\omega_{0}( & \left.T_{n-r}\left(\bigotimes_{j=1}^{r} Z_{c}^{(2)}\left(B_{j}\left(x_{j}\right) \otimes B_{r+j}\left(x_{r+j}\right)\right) \otimes \bigotimes_{s=2 r+1}^{n} B_{s}\left(x_{s}\right)\right)\right) \\
= & c^{r} t_{n-r}\left(\zeta\left(B_{1}, B_{r+1}\right), \ldots, \zeta\left(B_{r}, B_{2 r}\right), B_{2 r+1}, \ldots, B_{n}\right) \\
& \left(x_{1}-x_{n}, \ldots, x_{r}-x_{n}, x_{2 r+1}-x_{n}, \ldots\right) \\
& \cdot \prod_{j=1}^{r} \delta\left(x_{j}-x_{r+j}\right)
\end{aligned}
$$

for some $0 \leq r \leq n / 2$. Next note that for $B_{1}, B_{2} \in \mathcal{P}_{\text {hom }}$ it holds that $\zeta\left(B_{1}, B_{2}\right) \in \mathcal{P}_{\text {hom }}$ and that

$$
\operatorname{dim} \zeta\left(B_{1}, B_{2}\right) \leq \operatorname{dim} B_{1}+\operatorname{dim} B_{2}-4
$$

Using additionally the axiom (viii) Scaling Degree for $T$ and the formulas $\operatorname{sd} \delta\left(x_{j}-x_{r+j}\right)=4$ and $\operatorname{sd}\left(f_{1} \otimes f_{2}\right)=\operatorname{sd}\left(f_{1}\right)+\operatorname{sd}\left(f_{2}\right)$, we see that the scaling degree of the expression on the r.h.s. of (A.14) is bounded by

$$
\operatorname{sd}(\ldots) \leq \sum_{j=1}^{r} \operatorname{dim} \zeta\left(B_{j}, B_{j+r}\right)+\sum_{s=2 r+1}^{n} \operatorname{dim} B_{s}+4 r \leq \sum_{j=1}^{n} \operatorname{dim} B_{j} .
$$

\section{References}

[1] Bogoliubov, N.N., Shirkov, D.V.: Introduction to the Theory of Quantized Fields. Interscience Publishers, Geneva (1959)

[2] Brennecke, F., Dütsch, M.: Removal of violations of the Master Ward Identity in perturbative QFT. Rev. Math. Phys. 20, 119-172 (2008)

[3] Brunetti, R., Dütsch, M., Fredenhagen, K.: Perturbative algebraic quantum field theory and the renormalization groups. Adv. Theor. Math. Phys. 13, 1541-1599 (2009)

[4] Brunetti, R., Dütsch, M., Fredenhagen, K., Rejzner, K.: work in progress

[5] Buchholz, D., Fredenhagen, K.: A $C^{*}$-algebraic approach to interacting quantum field theories. Commun. Math. Phys. 377, 947-969 (2020)

[6] Dütsch, M.: "From Classical Field Theory to Perturbative Quantum Field Theory", Progress in Mathematical Physics 74, Birkhäuser, (2019)

[7] Dütsch, M., Boas, F.-M.: The Master Ward Identity. Rev. Math. Phys. 14, 9771049 (2002)

[8] Dütsch, M., Fredenhagen, K.: A local (perturbative) construction of observables in gauge theories: the example of QED. Commun. Math. Phys. 203, 71-105 (1999)

[9] Dütsch, M., Fredenhagen, K.: The Master Ward Identity and generalized Schwinger-Dyson equation in classical field theory. Commun. Math. Phys. 243, 275-314 (2003)

[10] Dütsch, M., Fredenhagen, K.: Causal perturbation theory in terms of retarded products, and a proof of the Action Ward Identity. Rev. Math. Phys. 16, 12911348 (2004) 
[11] Dütsch, M., Krahe, F., Scharf, G.: Scalar QED revisited. Nuovo Cimento A 106, 277-307 (1993)

[12] Epstein, H., Glaser, V.: The role of locality in perturbation theory. Ann. Inst. Henri Poincaré 19A, 211-295 (1973)

[13] Peters, L.: "The Master Ward Identity for the complex scalar field: From classical to quantum symmetries", Bachelor's Thesis, Göttingen University, (2020). arXiv:2103.05433

[14] Popineau, G., Stora, R.: A pedagogical remark on the main theorem of perturbative renormalization theory. Nucl. Phys. B 912, 70-78 (2016). (preprint: LAPP-TH, Lyon (1982))

[15] Rejzner, K.: "BV quantization in perturbative algebraic QFT: Fundamental concepts and perspectives", (2020). arXiv:2004.14272

[16] Tippner, F.: "Scalar QED with String-Localised Potentials", Bachelor's Thesis, Göttingen University, (2019)

Michael Dütsch, Luis Peters and Karl-Henning Rehren

Institute for Theoretical Physics

Georg-August University Göttingen

Friedrich-Hund-Platz 1

37077 Göttingen

Germany

e-mail: michael.duetsch@theorie.physik.uni-goettingen.de;

luis.peters@stud . uni-goettingen.de;

krehren@gwdg. de

Communicated by Christoph Kopper.

Received: August 19, 2020.

Accepted: March 19, 2021. 